Mathematical Medicine and Biology Page 1 of 40 doi:10.1093/imammb/dqnxxx

\title{
An evaluation of some assumptions underpinning the bidomain equations of electrophysiology
}

\author{
JONATHAN P WHITELEY \\ Department of Computer Science, University of Oxford, \\ Wolfson Building, Parks Road, Oxford OX1 3QD, UK.
}

[Received on]

\begin{abstract}
Tissue level cardiac electrophysiology is usually modelled by the bidomain equations, or the monodomain simplification of the bidomain equations. One assumption made when deriving the bidomain equations is that both the intracellular and extracellular space are in electrical equilibrium. This assumption neglects the disturbance of this equilibrium in thin regions close to the cell membrane known, as Debye layers. We first demonstrate that the governing equations at the cell, or microscale, level may be adapted to take account of these Debye layers with little additional complexity, provided the permittivity within the Debye layers satisfies certain conditions that are believed to be satisfied for biological cells. We then homogenise the microscale equations using a technique developed for an almost periodic microstructure. Cardiac tissue is usually modelled as sheets of cardiac fibres stacked on top of one another. A common assumption is that an orthogonal coordinate system can be defined at each point of cardiac tissue, where the first axis is in the fibre direction, the second axis is orthogonal to the first axis but lies in the sheet of cardiac fibres, and the third axis is orthogonal to the cardiac sheet. It is assumed further that both the intracellular and extracellular conductivity tensors are diagonal with respect to these axes, and that the diagonal entries of these tensors are constant across the whole tissue. Using the homogenisation technique we find that this assumption is usually valid for cardiac tissue, but highlight situations where the assumption may not be valid.
\end{abstract}

Keywords: Poisson-Nernst-Planck equations, cardiac, bidomain equations, homogenisation

\section{Introduction}

The bidomain equations, or their monodomain simplification, are commonly used to describe tissue level cardiac electrophysiology. As is the case for all mathematical models, several assumptions are made when deriving these equations. We begin by describing some of these assumptions.

At the cell level, cardiac tissue may be modelled as consisting of a fully connected intracellular space, a fully connected extracellular space, and a cell membrane separating these two regions. A first assumption usually made when deriving the bidomain equations is that both the intracellular space and extracellular space are in electrical equilibrium (Bruce et al., 2014; Hand and Griffith, 2010, 2011; Keener and Sneyd, 1998; Keener and Panfilov, 1996; Neu and Krassowska, 1993; Richardson and Chapman, 2011), which implies that the intracellular current density $\mathbf{j}_{i}$ and extracellular current density $\mathbf{j}_{e}$ are given by Ohm's law:

$$
\mathbf{j}_{i}=-\sigma_{i} \nabla \phi_{i}, \quad \mathbf{j}_{e}=-\sigma_{e} \nabla \phi_{e}
$$

where $\sigma_{i}, \sigma_{e}$ are the scalar conductivities of the intracellular and extracellular space, and $\phi_{i}, \phi_{e}$ are the potentials of intracellular and extracellular space. Although this is a good approximation in most of the intracellular and extracellular regions, it is not a good approximation in very thin layers in both spaces

(c) The author 2008. Published by Oxford University Press on behalf of the Institute of Mathematics and its Applications. All rights reserved. 
that are adjacent to the cell membrane, known as Debye layers; see, for example, Richardson (2009). To avoid explicitly taking account of these Debye layers, the cell membrane and the Debye layers on either side of the cell membrane are usually modelled as a capacitor of zero thickness, with surface charge density $Q$ given by

$$
Q=C_{m} V
$$

where $V$ is the potential difference across this capacitor, and $C_{m}$ is the capacitance (which is assumed to be constant). If we neglect the motion of cardiac tissue due to the heart beating, and use the capacitance assumption encapsulated by Eq. (1.1), homogenisation allows us to derive the familiar bidomain equations:

$$
\begin{aligned}
\nabla \cdot\left(\Sigma^{(i)} \nabla \Phi_{i}\right) & =\chi\left(C_{m} \frac{\partial V}{\partial t}+I_{m}\right) \\
\nabla \cdot\left(\Sigma^{(e)} \nabla \Phi_{e}\right) & =-\chi\left(C_{m} \frac{\partial V}{\partial t}+I_{m}\right) \\
V & =\Phi_{i}-\Phi_{e}
\end{aligned}
$$

where $\Phi_{i}, \Phi_{e}$ are the tissue level intracellular and extracellular potentials, $V$ is the transmembrane potential, $\Sigma^{(i)}, \Sigma^{(e)}$ are the intracellular and extracellular conductivity tensors, $\chi$ is the ratio of membrane surface area to total volume, and $I_{m}$ is the transmembrane current density flowing into the cell from extracellular space.

For the purpose of tissue level electrophysiological modelling, cardiac tissue is usually assumed to comprise sheets of cardiac fibres stacked on top of each other. An orthogonal coordinate system is defined at each point where the first axis lies in the direction of the cardiac fibre, the second axis lies in the sheet in which the fibre lies (but is orthogonal to the first axis), and the third axis is orthogonal to the sheet. It is then assumed that the both the intracellular and the extracellular conductivity tensors are diagonal with respect to these axes, with entries that are constant across cardiac tissue. Using this assumption, at each point in cardiac tissue a rotation matrix $P$ exists such that

$$
\Sigma^{(i)}=P^{\top}\left(\begin{array}{ccc}
\Sigma_{i, 1} & 0 & 0 \\
0 & \Sigma_{i, 2} & 0 \\
0 & 0 & \Sigma_{i, 3}
\end{array}\right) P, \quad \Sigma^{(e)}=P^{\top}\left(\begin{array}{ccc}
\Sigma_{e, 1} & 0 & 0 \\
0 & \Sigma_{e, 2} & 0 \\
0 & 0 & \Sigma_{e, 3}
\end{array}\right) P
$$

where constants $\Sigma_{i, k}, \Sigma_{e, k}$, for $k=1,2,3$, are the intracellular and extracellular conductivities in the direction of axis $k$ in the axes aligned with the tissue microstructure.

In this paper we investigate the validity of the assumptions described above. We begin by removing the assumption that intracellular space and extracellular space are in electrical equilibrium by explicitly modelling the ions present using the Poisson-Nernst-Planck (PNP) equations (Newman and ThomasAlyea, 2004). Richardson (2009) considered the similar problem of modelling the electrophysiology of the squid giant axon. Asymptotic analysis was used to derive a nonlinear capacitance relationship between charge density $Q$ and transmembrane potential $V$. Although a linear approximation to this relationship may be adequate for transmembrane potentials in the range normally seen, this model predicts that nonlinear effects may have an effect when the transmembrane potential is outside the normal range, such as defibrillation studies; see, for example, Bishop et al. (2007). The derivation of the nonlinear relationship between $Q$ and $V$ assumes that the permittivity of both intracellular space and extracellular space are constant, and that the cell membrane has a much lower permittivity than either of these two 
regions. A more physically detailed description of the permittivity (Cherepanov et al., 2003) takes account of a significantly lower permittivity in both the intracellular and extracellular space in the vicinity of the cell membrane. We extend the model described in Richardson (2009) to take account of this more detailed description of the permittivity of the intracellular and extracellular spaces, which allows further simplification of the governing equations. As a consequence, rather than asymptotic analysis yielding a nonlinear relationship between charge density $Q$ and transmembrane potential $V$, we obtain a linear approximation to the relationship between $Q$ and $V$ which is valid over a much larger range than previously realised.

Having derived a model of the electrical activity in the cell membrane and Debye layers, we then homogenise the governing equations in both the intracellular and extracellular spaces to derive a tissue level model of electrophysiology. Homogenisation of cardiac tissue usually assumes that the microstructure is periodic (Bruce et al., 2014; Hand and Griffith, 2010, 2011; Neu and Krassowska, 1993). This is, however, not the case for cardiac tissue where tissue level variations in fibre direction and sheet orientation result in a microstructure that is almost periodic, rather than periodic. We follow Richardson and Chapman (2011) who developed a technique for homogenising an almost periodic microstructure. This homogenisation technique allows the specification of tissue level intracellular and extracellular conductivity tensors when given a tissue level transformation between the almost periodic microstructure and a periodic microstructure. We may then compare the conductivity tensors derived from the homogenisation technique with those arising from making the assumptions that underpin Eq. (1.2).

The remainder of this paper is structured as follows. In Section 2 we write down the PNP equations in both the intracellular and extracellular space, and then non-dimensionalise these equations. We then exploit several pertinent limits in Section 3, allowing us to derive a model of electrical activity in the cell membrane and Debye layers. We then use homogenisation in Section 4 to derive tissue level bidomain equations, allowing the investigation of various assumptions on the conductivity tensors. Finally, in Section 5, we present our conclusions.

\section{The microscale mathematical model}

We partition the tissue into an intracellular region $\Omega_{i}$, an extracellular region $\Omega_{e}$, and a cell membrane region $\Omega_{m}$ that separates the intracellular region from the extracellular region. Detailed models exist for the transport of many individual ions across the cell membrane to form the transmembrane current; these are reviewed in Noble and Rudy (2001). Rather than use one of the more recent physiologically detailed models we will use the Beeler-Reuter model (Beeler and Reuter, 1977) to model the transmembrane current as comprising contributions only from sodium, potassium and calcium ions. By modelling only these ions we may explictly write down the governing equations concisely. The extension to more complex models follows naturally from that presented here. We note that these ions all have positive charge. Other ions, some with a negative charge, must be present to allow electroneutrality in both the intracellular and extracellular spaces away from the Debye layer. We follow Plonsey and Barr (2000) by making the assumptions that all other ions are monovalent negative ions, and the cell mebrane is impermeable to these ions.

We will make the further assumption that the radius of curvature of the cell membrane is much larger than both the width of the Debye layer, and the thickness of the cell membrane. We verify that this is indeed a valid assumptiuon in Section 3. Under these conditions, Richardson (2009) notes that the derivation of the governing equations may be streamlined by the use of regular Cartesian coordinates rather than general curvilinear coordinates. 


\subsection{The governing equations}

In the remainder of this study we use superscript asterisks to denote dimensional variables; variables without this superscript are the corresponding dimensionless variables.

2.1.1 Intracellular space. The Nernst-Planck equations governing the intracellular concentrations of the three ions $\mathrm{Na}^{+}, \mathrm{K}^{+}$and $\mathrm{Ca}^{2+}$, and the other monovalent negative ions, denoted by $N_{i}^{*}, K_{i}^{*}, C_{i}^{*}, A_{i}^{*}$, may be written, for $\mathbf{x}^{*} \in \Omega_{i}^{*}$ :

$$
\begin{aligned}
\frac{\partial N_{i}^{*}}{\partial t^{*}} & =\nabla^{*} \cdot\left(D_{N_{i}}^{*}\left(\nabla^{*} N_{i}^{*}+\frac{F}{R T} N_{i}^{*} \nabla^{*} \phi^{*}\right)\right), \\
\frac{\partial K_{i}^{*}}{\partial t^{*}} & =\nabla^{*} \cdot\left(D_{K_{i}}^{*}\left(\nabla^{*} K_{i}^{*}+\frac{F}{R T} K_{i}^{*} \nabla^{*} \phi^{*}\right)\right), \\
\frac{\partial C_{i}^{*}}{\partial t^{*}} & =\nabla^{*} \cdot\left(D_{C_{i}}^{*}\left(\nabla^{*} C_{i}^{*}+\frac{2 F}{R T} C_{i}^{*} \nabla^{*} \phi^{*}\right)\right), \\
\frac{\partial A_{i}^{*}}{\partial t^{*}} & =\nabla^{*} \cdot\left(D_{A_{i}}^{*}\left(\nabla^{*} A_{i}^{*}-\frac{F}{R T} A_{i}^{*} \nabla^{*} \phi^{*}\right)\right),
\end{aligned}
$$

where $\phi^{*}$ is the potential, $D_{N_{i}}^{*}, D_{K_{i}}^{*}, D_{C_{i}}^{*}, D_{A_{i}}^{*}$ are the diffusivities of sodium, potassium, calcium and other ions in intracellular space, $F$ is Faraday's constant, $R$ is the universal gas constant, $T$ is the absolute temperature, and $t^{*}$ is time. The electric potential satisfies Poisson's equation:

$$
-\nabla^{*} \cdot\left(\varepsilon^{*}\left(\mathbf{x}^{*}\right) \nabla^{*} \phi^{*}\right)=F\left(N_{i}^{*}+K_{i}^{*}+2 C_{i}^{*}-A_{i}^{*}\right),
$$

where $\varepsilon^{*}$ is the permittivity of intracellular space. As discussed earlier, we emphasise that we permit spatial variations in the permittivity and write $\varepsilon^{*}\left(\mathbf{x}^{*}\right)$, where $\mathbf{x}^{*}$ are the coordinates of a point in space.

2.1.2 Extracellular space. Using similar notation to that used in Eqs. (2.1)-(2.5) the Poisson-NernstPlanck equations in extracellular space are, for $\mathbf{x}^{*} \in \Omega_{e}^{*}$ :

$$
\begin{aligned}
\frac{\partial N_{e}^{*}}{\partial t^{*}} & =\nabla^{*} \cdot\left(D_{N_{e}}^{*}\left(\nabla^{*} N_{e}^{*}+\frac{F}{R T} N_{e}^{*} \nabla^{*} \phi^{*}\right)\right), \\
\frac{\partial K_{e}^{*}}{\partial t^{*}} & =\nabla^{*} \cdot\left(D_{K_{e}}^{*}\left(\nabla^{*} K_{e}^{*}+\frac{F}{R T} K_{e}^{*} \nabla^{*} \phi^{*}\right)\right), \\
\frac{\partial C_{e}^{*}}{\partial t^{*}} & =\nabla^{*} \cdot\left(D_{C_{e}}^{*}\left(\nabla^{*} C_{e}^{*}+\frac{2 F}{R T} C_{e}^{*} \nabla^{*} \phi^{*}\right)\right), \\
\frac{\partial A_{e}^{*}}{\partial t^{*}} & =\nabla^{*} \cdot\left(D_{A_{e}}^{*}\left(\nabla^{*} A_{e}^{*}-\frac{F}{R T} A_{e}^{*} \nabla^{*} \phi^{*}\right)\right), \\
-\nabla^{*} \cdot\left(\varepsilon^{*}\left(\mathbf{x}^{*}\right) \nabla^{*} \phi^{*}\right) & =F\left(N_{e}^{*}+K_{e}^{*}+2 C_{e}^{*}-A_{e}^{*}\right),
\end{aligned}
$$

where the subscript $e$ is used to represent extracellular space.

2.1.3 Cell membrane. In the cell membrane the electric potential satisfies, for $\mathbf{x}^{*} \in \Omega_{m}^{*}$ :

$$
-\nabla^{*} \cdot\left(\varepsilon^{*}\left(\mathbf{x}^{*}\right) \nabla^{*} \phi^{*}\right)=0 .
$$


We note that a surface charge on the inner leaflet of the membrane adjacent to the intracellular space is observed in many biological cells (Brown and Noble, 1978; Cherepanov et al., 2003; Elul, 1967; Kass and Krafte, 1987; Kell and DeFelice, 1988; Richardson, 2009). We include this term in the boundary conditions discussed in the next section.

2.1.4 Boundary conditions. Let $\mathbf{n}_{i}$ be the unit vector, normal to the interface between the intracellular space and the cell membrane and pointing from the intracellular space into the cell membrane, and $\mathbf{n}_{e}$ be the unit vector, normal to the interface between the extracellular space and the cell membrane and pointing from the extracellular space into the cell membrane. The assumption, stated in Section 2, that the radius of curvature of the cell membrane is much greater than the width of the cell membrane allows us to define a single unit normal vector $\mathbf{n}$, where

$$
\mathbf{n}=\mathbf{n}_{i}=-\mathbf{n}_{e} .
$$

Boundary conditions at the interface between the intracellular space and cell membrane are given by, for $\mathbf{x}^{*} \in \partial \Omega_{i}^{*} \cap \partial \Omega_{m}^{*}$ :

$$
\begin{aligned}
\left(-D_{N_{i}}^{*}\left(\nabla^{*} N_{i}^{*}+\frac{F}{R T} N_{i}^{*} \nabla^{*} \phi^{*}\right)\right) \cdot \mathbf{n} & =g_{N}^{*}, \\
\left(-D_{K_{i}}^{*}\left(\nabla^{*} K_{i}^{*}+\frac{F}{R T} K_{i}^{*} \nabla^{*} \phi^{*}\right)\right) \cdot \mathbf{n} & =g_{K}^{*}, \\
\left(-D_{C_{i}}^{*}\left(\nabla^{*} C_{i}^{*}+\frac{2 F}{R T} C_{i}^{*} \nabla^{*} \phi^{*}\right)\right) \cdot \mathbf{n} & =\frac{1}{2} g_{C}^{*}, \\
\left(-D_{A_{i}}^{*}\left(\nabla^{*} A_{i}^{*}-\frac{F}{R T} A_{i}^{*} \nabla^{*} \phi^{*}\right)\right) \cdot \mathbf{n} & =0, \\
{\left[\varepsilon^{*} \nabla^{*} \phi^{*}\right] \cdot \mathbf{n} } & =-f^{*}, \\
{\left[\phi^{*}\right] } & =0,
\end{aligned}
$$

where $g_{N}^{*}, g_{K}^{*}, g_{C}^{*}$ are the contributions to the ionic current density across the cell membrane into the intracellular space from sodium, potassium and calcium ions respectively (such as that described by Beeler and Reuter (1977)), $f^{*}$ denotes the surface charge density on the inner leaflet of the membrane adjacent to the intracellular space, and the jump of a quantity across the cell membrane boundary, [·], is defined to be the jump of a quantity from outside the membrane to inside the membrane. Similarly, at the interface between the extracellular space and cell membrane we have, for $\mathbf{x}^{*} \in \partial \Omega_{e}^{*} \cap \partial \Omega_{m}^{*}$ :

$$
\begin{aligned}
\left(-D_{N_{e}}^{*}\left(\nabla^{*} N_{e}^{*}+\frac{F}{R T} N_{e}^{*} \nabla^{*} \phi^{*}\right)\right) \cdot \mathbf{n} & =-g_{N}^{*}, \\
\left(-D_{K_{e}}^{*}\left(\nabla^{*} K_{e}^{*}+\frac{F}{R T} K_{e}^{*} \nabla^{*} \phi^{*}\right)\right) \cdot \mathbf{n} & =-g_{K}^{*}, \\
\left(-D_{C_{e}}^{*}\left(\nabla^{*} C_{e}^{*}+\frac{2 F}{R T} C_{e}^{*} \nabla^{*} \phi^{*}\right)\right) \cdot \mathbf{n} & =-\frac{1}{2} g_{C}^{*}, \\
\left(-D_{A_{e}}^{*}\left(\nabla^{*} A_{e}^{*}-\frac{F}{R T} A_{e}^{*} \nabla^{*} \phi^{*}\right)\right) \cdot \mathbf{n} & =0, \\
{\left[\varepsilon^{*} \nabla^{*} \phi^{*}\right] \cdot \mathbf{n} } & =0, \\
{\left[\phi^{*}\right] } & =0 .
\end{aligned}
$$


2.1.5 The permittivity. The permittivity is specified using the relationship proposed by Teschke et al. (2001), using parameters suitable for biological cells given by Cherepanov et al. (2003):

$$
\varepsilon^{*}\left(s^{*}\right)= \begin{cases}\varepsilon_{\mathrm{b}} \varepsilon_{0}\left(1+\left(\frac{\varepsilon_{\mathrm{b}}}{\varepsilon_{\mathrm{m}}}-1\right) \mathrm{e}^{-s^{*} / \lambda^{*}}\right)^{-1}, & \mathbf{x}^{*} \in \partial \Omega_{i}^{*}, \\ \varepsilon_{\mathrm{m}} \varepsilon_{0}, & \mathbf{x}^{*} \in \partial \Omega_{e}^{*}, \\ \mathbf{x}^{*} \in \partial \Omega_{m}^{*}, & \end{cases}
$$

where $\varepsilon_{\mathrm{m}}$ is the dielectric constant in the cell membrane, $\varepsilon_{\mathrm{b}}$ is the dielectric constant in the intracellular and extracellular spaces away from the cell membrane, $s^{*}$ is the distance from the cell membrane, and $\lambda^{*}$ is a constant. Following Cherepanov et al. (2003) we set $\varepsilon_{\mathrm{m}}=4$ (giving a permittivity in the cell membrane of $4 \varepsilon_{0}$, where $\varepsilon_{0}$ is the permittivity of free space), $\varepsilon_{\mathrm{b}}=78$, and $\lambda^{*}=10^{-9} \mathrm{~m}$. A plot of the permittivity described by Eq. (2.24) is given in Figure 1 .

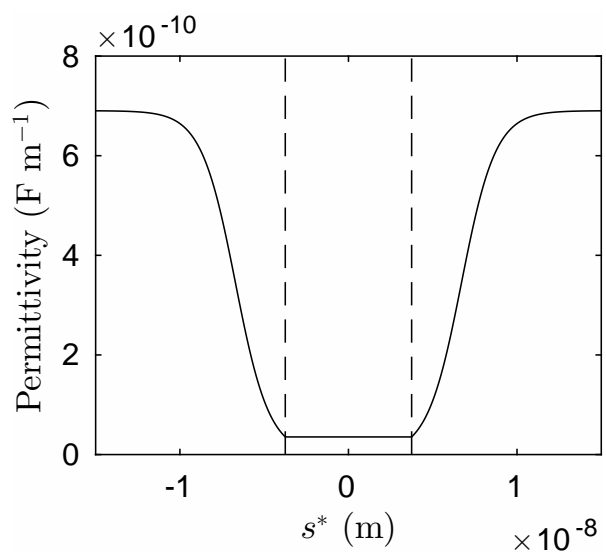

FIG. 1. The spatially varying permittivity. The dashed lines represent the boundaries of the membrane. Intracellular space lies to the right of the membrane, extracellular space to the left of the membrane.

\subsection{The dimensionless equations}

We use a similar nondimensionalisation to Richardson (2009):

$$
\begin{aligned}
& N_{i, e}^{*}=\Pi_{0} N_{i, e}, \quad K_{i, e}^{*}=\Pi_{0} K_{i, e}, \quad C_{i, e}^{*}=\Pi_{0} C_{i, e}, \quad A_{i, e}^{*}=\Pi_{0} A_{i, e}, \\
& \phi_{i, e}^{*}=\frac{R T}{F} \phi, \quad g_{N}^{*}=G g_{N}, \quad g_{K}^{*}=G g_{K}, \quad g_{C}^{*}=G g_{C}, \\
& D_{N_{i, e}}^{*}=\bar{D} D_{N_{i, e}}, \quad D_{K_{i, e}}^{*}=\bar{D} D_{K_{i, e}}, \quad D_{C_{i, e}}^{*}=\bar{D} D_{C_{i, e}}, \quad D_{A_{i, e}}^{*}=\bar{D} D_{A_{i, e}}, \\
& t^{*}=\tau t, \quad \mathbf{x}^{*}=L \mathbf{x}, \quad \varepsilon^{*}=\bar{\varepsilon} \varepsilon, \quad f^{*}=\frac{R T \bar{\varepsilon}}{F L \delta} f, \\
& \lambda^{*}=L \lambda, \quad s^{*}=L s,
\end{aligned}
$$

where $\tau$ is a representative timescale, $L$ is representative of the solution lengthscale, $\Pi_{0}$ is representative of an ion concentration, $\bar{D}$ is representative of the diffusivities of the ions present, $G$ is representative of 
the transmembrane ionic fluxes, $\bar{\varepsilon}$ is representative of the permittivity, and $\delta$ is a dimensionless parameter that will be defined later. The parameters given in Appenix A suggest choosing $\bar{D}=10^{-9} \mathrm{~m}^{2} \mathrm{~s}^{-1}$, $\Pi_{0}=140 \mathrm{~mol} \mathrm{~m}^{-3}$ and $R T / F=2.41 \times 10^{-2}$ V. Further, Eq. (2.24) suggests taking $\bar{\varepsilon}=\varepsilon_{\mathrm{b}} \varepsilon_{0}$; the value of $\varepsilon_{0}$ given in Appenix A then yields $\bar{\varepsilon}=6.9 \times 10^{-10} \mathrm{~F} \mathrm{~m}^{-1}$.

As explained earlier we model only the ions present in the electrophysiological model described by Beeler and Reuter (1977) to aid the clarity of this manuscript. When estimating the value of $G$ used in the nondimensionalisation we will, however, consider a more recent model (Luo and Rudy, 1994) to ensure applicability of the results presented here to more recent data. The fast sodium current is the transmembrane current with the largest magnitude for myocytes, and operates on the most rapid timescales. We therefore use this current when estimating a value for $G$, in contrast to Richardson (2009) who used the potassium current for this purpose when modelling the squid giant axon. We plot the fast sodium current density from the model described by Luo and Rudy (1994) in Figure 2. This figure suggests setting $G=3.5 \mathrm{~A} \mathrm{~m}^{-2}$. However, if we use this value of $G$ to estimate the natural timescale arising from the governing differential equations and boundary conditions, as used by Richardson (2009), we obtain

$$
\tau=\sqrt{\frac{\bar{\varepsilon} R T \Pi_{0}}{G^{2} F^{2}}} \approx 4.3 \times 10^{-3} \mathrm{~s},
$$

and see that this natural timescale is much slower than that on which the fast sodium current acts. Instead we set $\tau=10^{-3} \mathrm{~s}$ to give a more realistic timescale, and define

$$
G=\sqrt{\frac{\bar{\varepsilon} R T \Pi_{0}}{\tau^{2} F^{2}}}, \quad L=\frac{\bar{D} \Pi_{0}}{G}, \quad \delta=\sqrt{\frac{\bar{\varepsilon} R T}{L^{2} F^{2} \Pi_{0}}} .
$$

This yields $G=15.0 \mathrm{~A} \mathrm{~m}^{-2}, L=9.01 \times 10^{-4} \mathrm{~m}$, and $\delta=1.23 \times 10^{-6}$. The maximum modulus of the dimensionless fast sodium current density then takes the value $g_{N}=0.233$.

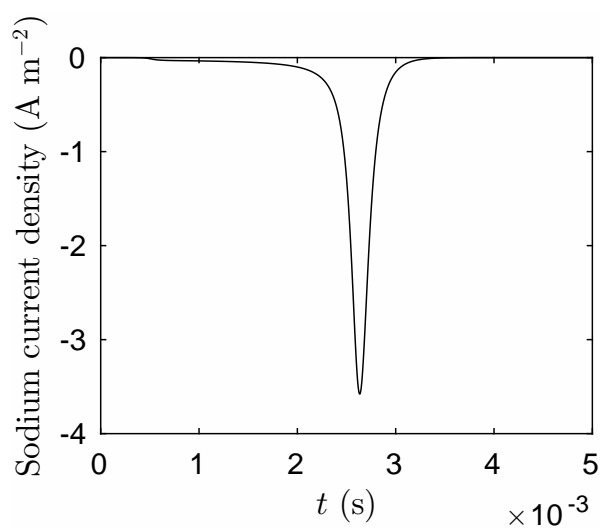

FIG. 2. The fast sodium current density from the electrophysiology model described by Luo and Rudy (1994).

The dimensional parameter that appears in the nondimensionalisation of the surface charge takes the value $R T \bar{\varepsilon} / F L \delta=1.5 \times 10^{-2} \mathrm{C} \mathrm{m}^{-2}$. Reported values of $f^{*}$ lie in the range between $-10^{-3} \mathrm{C} \mathrm{m}^{-2}$ and $-6 \times 10^{-2} \mathrm{C} \mathrm{m}^{-2}$, (Brown and Noble, 1978; Cherepanov et al., 2003; Elul, 1967; Kass and Krafte, 
1987; Kell and DeFelice, 1988), and so the dimensionless parameter $f$ lies in the range between approximately -0.06 and -4 . Further, the scaling used for the potential results in dimensionless potential differences across the alveolar membrane varying in the range between roughly -3 and 1 under normal circumstances.

Using Eqs. (2.1)-(2.5) we may write the dimensionless equations in the intracellular space as, for $\mathbf{x} \in \Omega_{i}$ :

$$
\begin{aligned}
\frac{\partial N_{i}}{\partial t} & =\delta \nabla \cdot\left(D_{N_{i}}\left(\nabla N_{i}+N_{i} \nabla \phi\right)\right), \\
\frac{\partial K_{i}}{\partial t} & =\delta \nabla \cdot\left(D_{K_{i}}\left(\nabla K_{i}+K_{i} \nabla \phi\right)\right), \\
\frac{\partial C_{i}}{\partial t} & =\delta \nabla \cdot\left(D_{C_{i}}\left(\nabla C_{i}+2 C_{i} \nabla \phi\right)\right), \\
\frac{\partial A_{i}}{\partial t} & =\delta \nabla \cdot\left(D_{A_{i}}\left(\nabla A_{i}-A_{i} \nabla \phi\right)\right), \\
-\nabla \cdot(\varepsilon \nabla \phi) & =\frac{1}{\delta^{2}}\left(N_{i}+K_{i}+2 C_{i}-A_{i}\right) .
\end{aligned}
$$

Similarly, we may use Eqs. (2.6)-(2.10) to obtain the dimensionless equations in the extracellular space. For $\mathbf{x} \in \Omega_{e}$ we have:

$$
\begin{aligned}
\frac{\partial N_{e}}{\partial t} & =\delta \nabla \cdot\left(D_{N_{e}}\left(\nabla N_{e}+N_{e} \nabla \phi\right)\right), \\
\frac{\partial K_{e}}{\partial t} & =\delta \nabla \cdot\left(D_{K_{e}}\left(\nabla K_{e}+K_{e} \nabla \phi\right)\right), \\
\frac{\partial C_{e}}{\partial t} & =\delta \nabla \cdot\left(D_{C_{e}}\left(\nabla C_{e}+2 C_{e} \nabla \phi\right)\right), \\
\frac{\partial A_{e}}{\partial t} & =\delta \nabla \cdot\left(D_{A_{e}}\left(\nabla A_{e}-A_{e} \nabla \phi\right)\right), \\
-\nabla \cdot(\varepsilon \nabla \phi) & =\frac{1}{\delta^{2}}\left(N_{e}+K_{e}+2 C_{e}-A_{e}\right) .
\end{aligned}
$$

In the cell mebrane, Eq. (2.11) allows us to write the dimensionless equation for the potential as, for $\mathbf{x} \in \Omega_{m}$ :

$$
-\nabla \cdot(\varepsilon \nabla \phi)=0
$$

We now write Eqs. (2.12)-(2.17) and Eqs. (2.18)-(2.23), the interface conditions at the interface between both intracellular and extracellular space with the cell membrane, in dimensionless form. These equations become, for $\mathbf{x} \in \partial \Omega_{i} \cap \partial \Omega_{m}$ :

$$
\begin{aligned}
\left(-D_{N_{i}}\left(\nabla N_{i}+N_{i} \nabla \phi\right)\right) \cdot \mathbf{n} & =g_{N}, \\
\left(-D_{K_{i}}\left(\nabla K_{i}+K_{i} \nabla \phi\right)\right) \cdot \mathbf{n} & =g_{K}, \\
\left(-D_{C_{i}}\left(\nabla C_{i}+2 C_{i} \nabla \phi\right)\right) \cdot \mathbf{n} & =\frac{1}{2} g_{C}, \\
\left(-D_{A_{i}}\left(\nabla A_{i}-A_{i} \nabla \phi\right)\right) \cdot \mathbf{n} & =0, \\
{[\varepsilon \nabla \phi] \cdot \mathbf{n} } & =-\frac{f}{\delta}, \\
{[\phi] } & =0,
\end{aligned}
$$


and for $\mathbf{x} \in \partial \Omega_{e} \cap \partial \Omega_{m}$ :

$$
\begin{aligned}
\left(-D_{N_{e}}\left(\nabla N_{e}+N_{e} \nabla \phi\right)\right) \cdot \mathbf{n} & =-g_{N}, \\
\left(-D_{K_{e}}\left(\nabla K_{e}+K_{e} \nabla \phi\right)\right) \cdot \mathbf{n} & =-g_{K}, \\
\left(-D_{C_{e}}\left(\nabla C_{e}+2 C_{e} \nabla \phi\right)\right) \cdot \mathbf{n} & =-\frac{1}{2} g_{C}, \\
\left(-D_{A_{e}}\left(\nabla A_{e}-A_{e} \nabla \phi\right)\right) \cdot \mathbf{n} & =0, \\
{[\varepsilon \nabla \phi] \cdot \mathbf{n} } & =0, \\
{[\phi] } & =0 .
\end{aligned}
$$

Finally, the dimensionless permittivity is given by

$$
\varepsilon(s)= \begin{cases}\left(1+\left(\frac{\varepsilon_{\mathrm{b}}}{\varepsilon_{\mathrm{m}}}-1\right) \mathrm{e}^{-s / \lambda}\right)^{-1}, & \mathbf{x} \in \partial \Omega_{i}, \quad \mathbf{x} \in \partial \Omega_{e}, \\ \frac{\varepsilon_{\mathrm{m}}}{\varepsilon_{\mathrm{b}}}, & \mathbf{x} \in \partial \Omega_{m},\end{cases}
$$

where $\lambda \approx 1.11 \times 10^{-6}$, and $s$ is the dimensionless distance to the membrane.

\section{The simplified microscale model}

The existence of the factor of $1 / \delta^{2}$ on the right hand side of both Eqs. (2.30) and (2.35) suggests that a boundary layer, of dimensionless width $\delta$, may exist in the solution of the governing equations for both the intracellular and extracellular regions, adjacent to the cell membrane. These boundary layers, known as Debye layers, are regions where ions are not in electrical equilibrium. A detailed derivation of this simplified model is given by Richardson (2009); we focus on the introduction of spatially varying permittivity in the Debye layers, and give only a summary of the derivation when it is identical to that given in Richardson (2009). We note that both the Debye layer thickness and cell membrane thickness are of $\mathscr{O}\left(10^{-9}\right) \mathrm{m}$; the assumption, stated in Section 2, that the radius of curvature of the cell membrane is much larger than these values is therefore not particularly restrictive.

The leading order solution of the microscale problem may be derived by considering three regions: (i) the intracellular space away from Debye layers; (ii) the extracellular space away from Debye layers; and (iii) a region comprising the Debye layers from both intracellular and extracellular space, and the cell membrane. We begin by deriving the solution in each of these regions, before matching these solutions.

\subsection{The outer solution in the intracellular region}

We write the solution in the outer region of intracellular space as a regular asymptotic expansion in powers of $\delta$ :

$$
\begin{aligned}
N_{i}^{(o)} & =N_{i, 0}^{(o)}+\delta N_{i, 1}^{(o)}+\ldots, & & K_{i}^{(o)}=K_{i, 0}^{(o)}+\delta K_{i, 1}^{(o)}+\ldots \\
C_{i}^{(o)} & =C_{i, 0}^{(o)}+\delta C_{i, 1}^{(o)}+\ldots, & & A_{i}^{(o)}=A_{i, 0}^{(o)}+\delta A_{i, 1}^{(o)}+\ldots \\
\phi & =\phi_{i, 0}^{(o)}+\delta \phi_{i, 1}^{(o)}+\ldots & &
\end{aligned}
$$


Substituting these asymptotic expansions into Eqs. (2.26)-(2.30), the leading order terms imply

$$
\begin{array}{ll}
N_{i, 0}^{(o)}=\bar{N}_{i}, & K_{i, 0}^{(o)}=\bar{K}_{i}, \quad C_{i, 0}^{(o)}=\bar{C}_{i}, \\
A_{i, 0}^{(o)}=\bar{A}_{i}, & \bar{N}_{i}+\bar{K}_{i}+2 \bar{C}_{i}-\bar{A}_{i}=0,
\end{array}
$$

where $\bar{N}_{i}, \bar{K}_{i}, \bar{C}_{i}, \bar{A}_{i}$ are constants, and denote the concentrations $N_{i}, K_{i}, C_{i}, A_{i}$ away from the Debye layer.

Terms in Eqs. (2.26)-(2.30) of $\mathscr{O}(\delta)$ then give

$$
\begin{aligned}
\frac{\partial N_{i, 1}^{(o)}}{\partial t} & =\nabla \cdot\left(D_{N_{i}} \bar{N}_{i} \nabla \phi_{i, 0}^{(o)}\right), \\
\frac{\partial K_{i, 1}^{(o)}}{\partial t} & =\nabla \cdot\left(D_{K_{i}} \bar{K}_{i} \nabla \phi_{i, 0}^{(o)}\right), \\
\frac{\partial C_{i, 1}^{(o)}}{\partial t} & =\nabla \cdot\left(2 D_{C_{i}} \bar{C}_{i} \nabla \phi_{i, 0}^{(o)}\right), \\
\frac{\partial A_{i, 1}^{(o)}}{\partial t} & =-\nabla \cdot\left(D_{A_{i}} \bar{A}_{i} \nabla \phi_{i, 0}^{(o)}\right), \\
N_{i, 1}^{(o)}+K_{i, 1}^{(o)}+2 C_{i, 1}^{(o)}-A_{i, 1}^{(o)} & =0 .
\end{aligned}
$$

Summing Eqs. (3.3) \& (3.4) with twice Eq. (3.5), subtracting Eq. (3.6), and then using Eq. (3.7) gives

$$
\nabla^{2} \phi_{i, 0}^{(o)}=0 .
$$

Using Eqs. (3.3)-(3.7) we may deduce that the leading order dimensionless intracellular current density, $\mathbf{j}_{i, 0}$, is given in terms of the dimensionless intracellular conductivity, $\sigma_{i}$, by:

$$
\begin{aligned}
\mathbf{j}_{i, 0} & =-\sigma_{i} \nabla \phi_{i, 0}^{(o)}, \\
\text { where } \sigma_{i} & =D_{N_{i}} \bar{N}_{i}+D_{K_{i}} \bar{K}_{i}+4 D_{C_{i}} \bar{C}_{i}+D_{A_{i}} \bar{A}_{i},
\end{aligned}
$$

giving Ohm's law at leading order. Using the nondimensionalisation

$$
\mathbf{j}_{i, 0}^{*}=G \mathbf{j}_{i, 0}, \quad \sigma_{i}^{*}=\bar{\sigma} \sigma_{i},
$$

the nondimensionalisation in Section 2.2 suggests choosing

$$
\bar{\sigma}=\frac{G L F}{R T} \approx 0.56 \mathrm{~S} \mathrm{~m}^{-1}
$$

resulting in dimensional conductivities in line with those reported elsewhere (Clerc, 1976; Sommer, 1983).

\subsection{The outer solution in the extracellular region}

The outer solution in the extracellular region may be derived in a similar fashion to the solution in the intracellular region. Using notation that follows naturally from that introduced in Section 3.1, the leading order terms from Eqs. (2.31)-(2.35) give

$$
\begin{array}{ll}
N_{e, 0}^{(o)}=\bar{N}_{e}, & K_{e, 0}^{(o)}=\bar{K}_{i e}, \quad C_{e, 0}^{(o)}=\bar{C}_{e}, \\
A_{e, 0}^{(o)}=\bar{A}_{e}, & \bar{N}_{e}+\bar{K}_{e}+2 \bar{C}_{e}-\bar{A}_{e}=0,
\end{array}
$$


where $\bar{N}_{e}, \bar{K}_{e}, \bar{C}_{e}, \bar{A}_{e}$ are constants, and denote the concentrations $N_{e}, K_{e}, C_{e}, A_{e}$ away from the Debye layer (which may be different to those in intracellular space). As with the outer solution in the intracellular region, terms of $\mathscr{O}(\delta)$ then imply

$$
\nabla^{2} \phi_{e, 0}^{(o)}=0
$$

and that the leading order current flux $\mathbf{j}_{e, 0}$ satisfies Ohm's law:

$$
\begin{aligned}
\mathbf{j}_{e, 0} & =-\sigma_{e} \nabla \phi_{e, 0}^{(o)} \\
\text { for } \sigma_{e} & =D_{N_{e}} \bar{N}_{e}+D_{K_{e}} \bar{K}_{e}+4 D_{C_{e}} \bar{C}_{e}+D_{A_{e}} \bar{A}_{e}
\end{aligned}
$$

where $\sigma_{e}$ is the dimensionless extracellular conductivity.

\subsection{The solution in the Debye layers and cell membrane}

As noted by Richardson (2009), making the assumption that the radius of curvature of the membrane is much larger than the width of the Debye layer allows us choose coordinates where the vector $\mathbf{n}$ points along the negative $x$-axis, and to approximate the membrane as occupying the region $-\delta a<x<\delta a$, with the Debye layer in the intracellular space occupying $x>\delta a$, and the Debye layer in the extracellular space occupying $x<-\delta a$. The presence of the term in $1 / \delta^{2}$ on the right hand side of Eqs. (2.30) and (2.35) suggests rescaling the $x$-coordinate in the Debye layers and cell membrane by writing

$$
x=\delta X \text {. }
$$

The membrane now occupies the region $-a<X<a$. Using the parameters given in Table 1 we take $a=3.4$ dimensionless units. On using Eq. (2.49) we see that permittivity in the cell membrane and Debye layers is given by

$$
\varepsilon(X)= \begin{cases}\left(1+\left(\frac{\varepsilon_{\mathrm{b}}}{\varepsilon_{\mathrm{m}}}-1\right) \mathrm{e}^{-(X-a) / \Lambda}\right)^{-1}, & X>a, \\ \frac{\varepsilon_{\mathrm{m}}}{\varepsilon_{\mathrm{b}}}, & -a<X<a, \\ \left(1+\left(\frac{\varepsilon_{\mathrm{b}}}{\varepsilon_{\mathrm{m}}}-1\right) \mathrm{e}^{(X+a) / \Lambda}\right)^{-1}, & X<-a,\end{cases}
$$

where $\Lambda=\lambda / \delta \approx 0.9$.

We now describe the solution in the Debye layers and cell membrane, before using the interface conditions Eqs. (2.37)-(2.48), re-scaled using Eq. (3.14), to generate a relationship between the outer solutions and the solutions in the Debye layers.

3.3.1 The Debye layer solution in the intracellular region. We write the solution in the Debye region of intracellular space as an asymptotic solution in powers of $\delta$ :

$$
\begin{array}{rlrl}
N_{i}^{(D)} & =N_{i, 0}^{(D)}+\delta N_{i, 1}^{(D)}+\ldots, & & K_{i}^{(D)}=K_{i, 0}^{(D)}+\delta K_{i, 1}^{(D)}+\ldots, \\
C_{i}^{(D)} & =C_{i, 0}^{(D)}+\delta C_{i, 1}^{(D)}+\ldots, & A_{i}^{(D)}=A_{i, 0}^{(D)}+\delta A_{i, 1}^{(D)}+\ldots, \quad j=1,2, \ldots, M, \\
\phi & =\phi_{i, 0}^{(D)}+\delta \phi_{i, 1}^{(D)}+\ldots & &
\end{array}
$$


Using the rescaling given by Eq. (3.14), the leading order terms in Eqs. (2.26)-(2.30) are:

$$
\begin{aligned}
\frac{\partial N_{i, 0}^{(D)}}{\partial X}+N_{i, 0}^{(D)} \frac{\partial \phi_{i, 0}^{(D)}}{\partial X} & =0 \\
\frac{\partial K_{i, 0}^{(D)}}{\partial X}+K_{i, 0}^{(D)} \frac{\partial \phi_{i, 0}^{(D)}}{\partial X} & =0 \\
\frac{\partial C_{i, 0}^{(D)}}{\partial X}+2 C_{i, 0}^{(D)} \frac{\partial \phi_{i, 0}^{(D)}}{\partial X} & =0 \\
\frac{\partial A_{i, 0}^{(D)}}{\partial X}-A_{i, 0}^{(D)} \frac{\partial \phi_{i, 0}^{(D)}}{\partial X} & =0 \\
-\frac{\partial}{\partial X}\left(\varepsilon(X) \frac{\partial \phi_{i, 0}^{(D)}}{\partial X}\right) & =N_{i, 0}^{(D)}+K_{i, 0}^{(D)}+2 C_{i, 0}^{(D)}-A_{i, 0}^{(D)} .
\end{aligned}
$$

The solutions to Eqs. (3.17)-(3.20), satisfying the condition that these solutions match the outer solution given by Eqs. (3.1) and (3.2) as $X \rightarrow \infty$, are given by

$$
\begin{aligned}
N_{i, 0}^{(D)} & =\bar{N}_{i} \exp \left(\phi_{i, 0}^{(o)}-\phi_{i, 0}^{(D)}\right) \\
K_{i, 0}^{(D)} & =\bar{K}_{i} \exp \left(\phi_{i, 0}^{(o)}-\phi_{i, 0}^{(D)}\right) \\
C_{i, 0}^{(D)} & =\bar{C}_{i} \exp \left(2\left(\phi_{i, 0}^{(o)}-\phi_{i, 0}^{(D)}\right)\right) \\
A_{i, 0}^{(D)} & =\bar{A}_{i} \exp \left(\phi_{i, 0}^{(D)}-\phi_{i, 0}^{(o)}\right) .
\end{aligned}
$$

We note that these solutions do not yet satisfy the conditions at the interface between the Debye layer and the cell membrane, and will return to this point when matching the outer solution with the Debye layer solution in Section 3.6.

3.3.2 The Debye layer solution in the extracellular region. Using notation for the extracellular region that follows from that used in the intracellular region, and a similar procedure to that used in Section 3.3.1, we may deduce that the leading order potential in the Debye layer satisfies:

$$
-\frac{\partial}{\partial X}\left(\varepsilon(X) \frac{\partial \phi_{e, 0}^{(D)}}{\partial X}\right)=N_{e, 0}^{(D)}+K_{e, 0}^{(D)}+2 C_{e, 0}^{(D)}-A_{e, 0}^{(D)},
$$

where

$$
\begin{aligned}
N_{e, 0}^{(D)} & =\bar{N}_{e} \exp \left(\phi_{e, 0}^{(o)}-\phi_{e, 0}^{(D)}\right) \\
K_{e, 0}^{(D)} & =\bar{K}_{e} \exp \left(\phi_{e, 0}^{(o)}-\phi_{e, 0}^{(D)}\right) \\
C_{e, 0}^{(D)} & =\bar{C}_{e} \exp \left(2\left(\phi_{e, 0}^{(o)}-\phi_{e, 0}^{(D)}\right)\right) \\
A_{e, 0}^{(D)} & =\bar{A}_{e} \exp \left(\phi_{e, 0}^{(D)}-\phi_{e, 0}^{(o)}\right) .
\end{aligned}
$$


Similar remarks apply to these solutions as were made on the solution in the Debye layer in the intracellular region: the solutions given by Eqs. (3.27)-(3.30) satisfy the matching conditions with the outer solutions in the extracellular space as $X \rightarrow-\infty$, but are not yet guaranteed to satisfy the boundary conditions at the cell membrane.

3.3.3 The solution in the cell membrane. Using the rescaling given by Eq. (3.14), the leading order potential in the cell membrane satisfies

$$
-\frac{\partial}{\partial X}\left(\frac{\varepsilon_{\mathrm{m}}}{\varepsilon_{\mathrm{b}}} \frac{\partial \phi_{0}^{(M)}}{\partial X}\right)=0 .
$$

3.3.4 Conditions at the boundaries of the membrane. At the interface between the intracellular space and the cell membrane, and between the extracellular space and the cell membrane, Eqs. (2.41), (2.42), (2.47) and (2.48) give the following conditions for the leading order potential:

$$
\begin{aligned}
\left.\phi_{i, 0}^{(D)}\right|_{X=a} & =\left.\phi_{0}^{(M)}\right|_{X=a}, \\
\left.\phi_{e, 0}^{(D)}\right|_{X=-a} & =\left.\phi_{0}^{(M)}\right|_{X=-a}, \\
\left.\frac{\varepsilon_{\mathrm{m}}}{\varepsilon_{\mathrm{b}}} \frac{\partial \phi_{i, 0}^{(D)}}{\partial X}\right|_{X=a} & =\left.\frac{\varepsilon_{\mathrm{m}}}{\varepsilon_{\mathrm{b}}} \frac{\partial \phi_{0}^{(M)}}{\partial X}\right|_{X=a}-f \\
\left.\frac{\varepsilon_{\mathrm{m}}}{\varepsilon_{\mathrm{b}}} \frac{\partial \phi_{e, 0}^{(D)}}{\partial X}\right|_{X=-a} & =\left.\frac{\varepsilon_{\mathrm{m}}}{\varepsilon_{\mathrm{b}}} \frac{\partial \phi_{0}^{(M)}}{\partial X}\right|_{X=-a} .
\end{aligned}
$$

3.3.5 An approximate analytic solution in the Debye layers and cell membrane. We now derive an explicit approximate solution for the leading order potential in the Debye layers and cell membrane, which we later verify by comparison with the numerical solution to the full system of governing equations. Writing

$$
M_{i}=\left.\phi_{i, 0}^{(D)}\right|_{X=a}, \quad M_{e}=\left.\phi_{e, 0}^{(D)}\right|_{X=-a}
$$

the solution to Eq. (3.31), that satisfies the continuity conditions at the boundaries of the membrane given by Eqs. (3.32) and (3.33) is, for $-a<X<a$,

$$
\phi_{0}^{(M)}=\frac{M_{i}+M_{e}}{2}+\frac{M_{i}-M_{e}}{2 a} X .
$$

Recalling the typical values for $\varepsilon_{\mathrm{m}}, \varepsilon_{\mathrm{b}}, f$ given in Sections 2.1.5 and 2.2, and solution for $\phi_{0}^{(M)}$ given by Eq. (3.37), we see that the dimensionless surface charge on the inner leaflet of the membrane adjacent to the intracellular space is normally such that

$$
\left|\frac{\varepsilon_{\mathrm{m}}}{\varepsilon_{\mathrm{b}}} \frac{\partial \phi_{0}^{(M)}}{\partial X}\right| \ll|f|,
$$


and so we may approximate the boundary condition Eq. (3.34) by

$$
\left.\frac{\partial \phi_{i, 0}^{(D)}}{\partial X}\right|_{X=a}=-\frac{\varepsilon_{\mathrm{b}} f}{\varepsilon_{\mathrm{m}}} .
$$

We may then solve Eq. (3.21) for $\phi_{i, 0}^{(D)}$ subject to the boundary condition Eq. (3.38) at the cell membrane that is now independent of the potential in the cell membrane, i.e. both the differential equation and boundary conditions satisfied by $\phi_{i, 0}^{(D)}$ are independent of $\phi_{0}^{(M)}$.

Using the parameters given in Table 1 we see that $C_{i}^{*} \ll N_{i}^{*}, K_{i}^{*}$. The nondimensionalisation given by Eq. (2.25) then allows us to make the approximation that $C_{i}=0$. Furthermore, if we are sufficiently close to the cell membrane, the intracellular permittivity given by Eq. (3.15) may be approximated by $\varepsilon=\frac{\varepsilon_{\mathrm{m}}}{\varepsilon_{\mathrm{b}}}$. Using these approximations Eq. (3.21) becomes, on using Eqs. (3.2) and (3.22)-(3.25),

$$
-\frac{\partial^{2} \phi_{i, 0}^{(D)}}{\partial X^{\prime 2}}=\frac{2 \varepsilon_{\mathrm{b}}}{\varepsilon_{\mathrm{m}}}\left(\bar{N}_{i}+\bar{K}_{i}\right) \sinh \left(\phi_{i, 0}^{(o)}-\phi_{i, 0}^{(D)}\right),
$$

with solution, satisfying the boundary condition at $X=a$ given by Eq. (3.38),

$$
\begin{aligned}
& \phi_{i, 0}^{(D)}= \\
& \phi_{i, 0}^{(o)}+2 \log \left(\tanh \left(\sqrt{\frac{\varepsilon_{\mathrm{b}}\left(\bar{N}_{i}+\bar{K}_{i}\right)}{2 \varepsilon_{\mathrm{m}}}}(X-a)+\frac{1}{2} \sinh ^{-1}\left(\frac{4}{-f} \sqrt{\frac{\varepsilon_{\mathrm{m}}\left(\bar{N}_{i}+\bar{K}_{i}\right)}{2 \varepsilon_{\mathrm{b}}}}\right)\right)\right) .
\end{aligned}
$$

The magnitude of the logarithmic term on the right hand side of this expression decays rapidly away from $X=a$, with the magnitude of this term being less than 0.01 for $X>a+0.01$; it is valid to use the approximation $\varepsilon=\frac{\varepsilon_{\mathrm{m}}}{\varepsilon_{\mathrm{b}}}$ on this interval. For $X>a+0.01$ we may approximate Eq. (3.39) by $\phi_{i, 0}^{(D)}=\phi_{i, 0}^{(o)}$, which satisfies Eq. (3.21), as $\phi_{i, 0}^{(o)}$ is independent of $X$. The solution given by Eq. (3.39) is therefore valid in the whole Debye layer and satisfies both the boundary condition Eq. (3.38) and the matching condition with the outer solution. We observe that Eq. (3.39) gives

$$
M_{i}=\phi_{i, 0}^{(o)}+2 \log \left(\tanh \left(\frac{1}{2} \sinh ^{-1}\left(\frac{4}{-f} \sqrt{\frac{\varepsilon_{\mathrm{m}}\left(\bar{N}_{i}+\bar{K}_{i}\right)}{2 \varepsilon_{\mathrm{b}}}}\right)\right)\right),
$$

indicating that there is a fixed difference between the intracellular potential at the interface with the cell membrane, and the outer solution for the intracellular potential. We note further that the solution for $\phi_{i, 0}^{(D)}$ given by Eq. (3.39) varies over a dimensional lengthscale of $10^{-10} \mathrm{~m}$. This lengthscale is comparable to the size of ions, and so it is possible that the continuum model described by partial differential equations does not hold. We discuss this further in Section 5.

Using a similar argument the solution for $\phi_{e, 0}^{(D)}$ is given by

$$
\phi_{e, 0}^{(D)}=\phi_{e, 0}^{(o)}-2 \log \left(\tanh \left(B-\sqrt{\frac{\varepsilon_{\mathrm{b}}\left(\bar{N}_{i}+\bar{K}_{i}\right)}{2 \varepsilon_{\mathrm{m}}}}(X+a)\right)\right),
$$


where $M_{e}$ and $B$ are specified by the boundary conditions Eqs. (3.33) and (3.35):

$$
\begin{aligned}
\sqrt{\frac{\varepsilon_{\mathrm{m}}}{8 a^{2} \varepsilon_{\mathrm{b}}\left(\bar{N}_{i}+\bar{K}_{i}\right)}}\left(M_{e}-M_{i}\right) & =1-\mathrm{e}^{\left(M_{e}-\phi_{e}^{(o)}\right) / 2}, \\
B & =\tanh ^{-1} \mathrm{e}^{\left(\phi_{e}^{(o)}-M_{e}\right) / 2} .
\end{aligned}
$$

Noting that

$$
\sqrt{\frac{\varepsilon_{\mathrm{m}}}{8 a^{2} \varepsilon_{\mathrm{b}}\left(\bar{N}_{i}+\bar{K}_{i}\right)}}=\mathscr{O}\left(10^{-5}\right)
$$

we see that an approximate solution for $\phi_{e, 0}^{(D)}$ and an expression for the constant $M_{e}$ are given by

$$
\begin{aligned}
\phi_{e, 0}^{(D)} & =\phi_{e, 0}^{(o)}, \\
M_{e} & =\phi_{e, 0}^{(o)},
\end{aligned}
$$

indicating that there is no Debye layer in extracellular space.

We now investigate the accuracy of the approximate solutions in both Debye layers and the cell membrane, given by Eqs. (3.37), (3.39) and (3.43), by comparing these approximate solutions to the numerical solution of Eqs. (3.21), (3.26) and (3.31), subject to the boundary conditions given by Eqs. (3.32)(3.35). The numerical solution was calculated in two steps. First, for given values of $M_{i}$ and $M_{e}$ (that are initially unknown), we may solve Eqs. (3.21), (3.26) and (3.31), subject to the two boundary conditions given by Eqs. (3.32)-(3.33) and also to matching with the outer solutions as $X \rightarrow \pm \infty$, by using the finite difference method. The second step is to find the two values $M_{i}$ and $M_{e}$ so that the finite difference solution calculated satisfies the two remaining boundary conditions, given by Eqs. (3.34)-(3.35). This second step requires the solution of two simultaneous nonlinear algebraic equations, and was achieved by using Newton's method with numerical approximations to the derivatives. The mesh used for the finite difference method was chosen to ensure that doubling the number of nodes in the mesh would change the solution for $\phi$ at each node by less than the specified tolerance of $10^{-5}$.

We set the dimensionless charge on the inner leaflet of the cell membrane, $f$, to -1.5. The dimensionless transmembrane potential typically varies in the range from -3 to 1 . In the left hand column of Figure 3 we set $\phi_{e, 0}^{(o)}=0, \phi_{i, 0}^{(o)}=1$, giving a dimensionless transmembrane potential (based on the outer solutions for the potential) of 1 . The plots in the right hand column show the corresponding plots when $\phi_{e, 0}^{(o)}=0, \phi_{i, 0}^{(o)}=-3$, giving a dimensionless transmembrane potential (based on the outer solutions for the potential) of -3 . Plots (a) and (b) show the solution in both Debye layers and the cell membrane. Solid lines represent the numerical solution of the full system of equations, broken lines represent the approximate analytic solution, and dotted lines indicate the location of the boundaries of the cell membrane. In plots (c) and (d) we expand the solution in the intracellular Debye layer and cell membrane in the vicinity of the boundary between these regions. Plots (e) and (f) show the solution in the extracellular Debye layer and cell membrane in the vicinity of the boundary between these regions. It is apparent that the approximate analytic solution is a very good approximation to the numerical solution of the full system of equations. In plots (e) and (f) we note that the numerical solution in the vicinity of the boundary between the cell membrane and extracellular space is much smoother than the analytical approximation. This is due to the approximation used when deriving the approximate analytic solution given by Eqs. (3.43) and (3.44); the resulting approximation of constant extracellular potential in the 
Debye layer will be unable to satisfy exactly the boundary condition given by Eq. (3.35). By contrast the numerical solution does satisfy this boundary condition and will therefore exhibit a smoother solution.

\subsection{The transmembrane potential}

Due to the presence of Debye layers, we need to be precise on our definition of the transmembrane potential. We define the transmembrane potential, $V_{m}$, to be the difference in leading order potential across the membrane, i.e.

$$
V_{m}=\left.\phi_{i, 0}^{(D)}\right|_{X=a}-\left.\phi_{e, 0}^{(D)}\right|_{X=-a} .
$$

Using the approximate analytic solution for the leading order potential given by Eqs. (3.36), (3.40) and (3.44), we obtain

$$
\begin{aligned}
V_{m} & =M_{i}-M_{e} \\
& =\phi_{i, 0}^{(o)}-\phi_{e, 0}^{(o)}+2 \log \left(\tanh \left(\frac{1}{2} \sinh ^{-1}\left(\frac{4}{-f} \sqrt{\frac{\varepsilon_{\mathrm{m}}\left(\bar{N}_{i}+\bar{K}_{i}\right)}{2 \varepsilon_{\mathrm{b}}}}\right)\right)\right) \\
& =V_{m}^{(o)}+2 \log \left(\tanh \left(\frac{1}{2} \sinh ^{-1}\left(\frac{4}{-f} \sqrt{\frac{\varepsilon_{\mathrm{m}}\left(\bar{N}_{i}+\bar{K}_{i}\right)}{2 \varepsilon_{\mathrm{b}}}}\right)\right)\right),
\end{aligned}
$$

where

$$
V_{m}^{(o)}=\phi_{i, 0}^{(o)}-\phi_{e, 0}^{(o)}
$$

We see that, when using the approximate solution derived here, $V_{m}$ and $V_{m}^{(o)}$ differ by a constant value.

We now verify the relationship given by Eq. (3.47), for the range of values of $f$ given in Section 2.2. In Figure 4(a) we plot the relationship between $V_{m}^{(o)}$ and $V_{m}$, where solid lines represent the solution of Eqs. (3.21), (3.26) and (3.31), subject to the boundary conditions given by Eqs. (3.32)-(3.35), and broken lines represent the approximation given by Eq. (3.47). We use three values of $f: f=-0.06$ (the top pair of lines); $f=-1.5$ (the middle pair of lines); and $f=-4$ (the bottom pair of lines). We noted earlier that $V_{m}$ typically varies in the range between -3 and 1 . We see in Figure 4(a) that Eq. (3.47) is an excellent approximation to the solution of the full system of equations for a wide range of $V_{m}$, including those typically seen, and also for the range of values of $f$ believed to occur.

\subsection{The surface charge density on the membrane}

When matching the outer solutions with the Debye layer solutions we will see that it is convenient to work with the charge density on the membrane. We define $Q$ to be the leading order dimensionless surface charge density per unit dimensionless area of membrane in the Debye layer at $X=a$. Integrating Eq. (3.21) across the Debye layer, using the boundary condition given by Eq. (3.34), and the matching condition with the outer solution gives

$$
Q=\frac{\varepsilon_{\mathrm{m}}}{\varepsilon_{\mathrm{b}}} \frac{\partial \phi^{(M)}}{\partial X}=f+\int_{a}^{\infty} N_{i, 0}^{(D)}+K_{i, 0}^{(D)}+2 C_{i, 0}^{(D)}-A_{i, 0}^{(D)} \mathrm{d} X .
$$




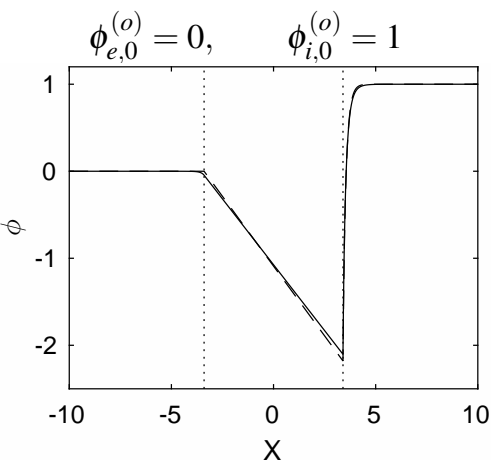

(a)

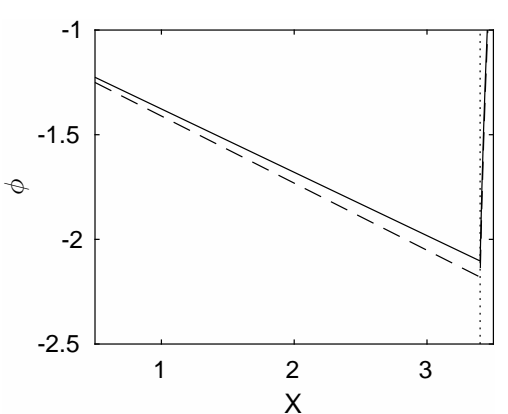

(c)

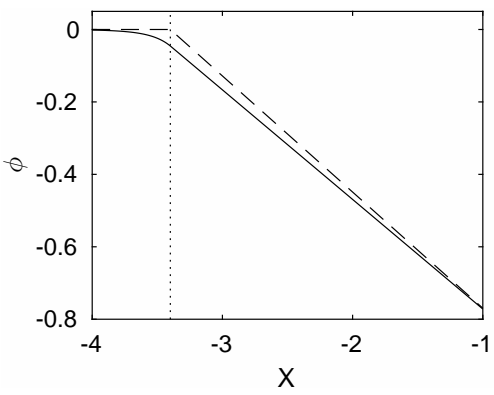

(e)

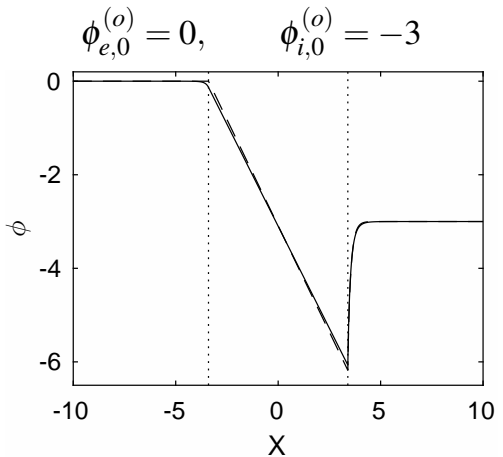

(b)

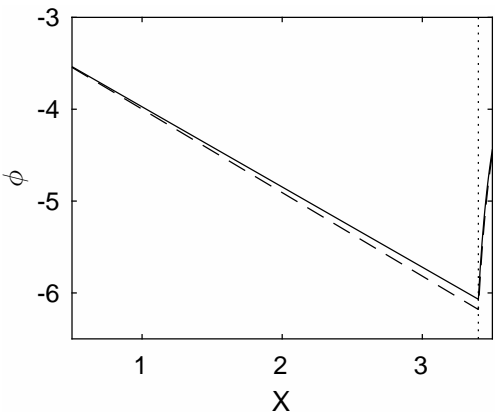

(d)

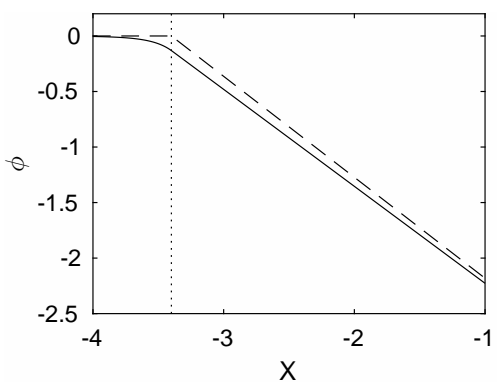

(f)

FIG. 3. The solution for the dimensionless potential in the cell membrane and Debye layers. The solid lines represent the numerical solution of Eqs. (3.21), (3.26) and (3.31), subject to the boundary conditions given by Eqs. (3.32)-(3.35), and the dashed lines represent the analytical approximations given by Eqs. (3.37), (3.39) and (3.43). Dotted lines represent the boundaries of the membrane. In the left hand column $\phi_{e, 0}^{(o)}=0, \phi_{i, 0}^{(o)}=1$, in the right hand column $\phi_{e, 0}^{(o)}=0, \phi_{i, 0}^{(o)}=-3$. Plots (a) and (b) show the solutions in both Debye layers and the cell membrane. Plots (c) and (d) show the solutions in the vicinity of the boundary between the intracellular Debye layer and cell membrane. Plots (e) and (f) show the solutions in the vicinity of the boundary between the extracellular Debye layer and cell membrane.

Similarly, using Eqs. (3.26) and (3.35) we may deduce that the leading order dimensionless surface charge density per unit dimensionless area of membrane in the Debye layer at $X=-a$ is given by $-Q$, 


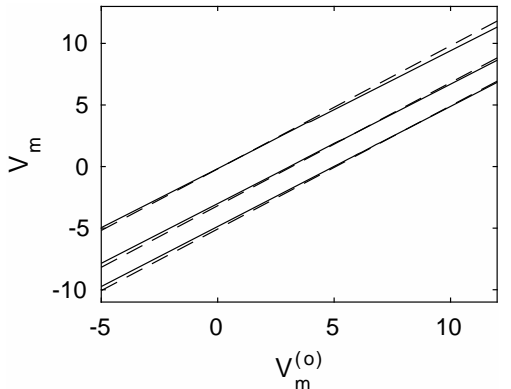

(a)

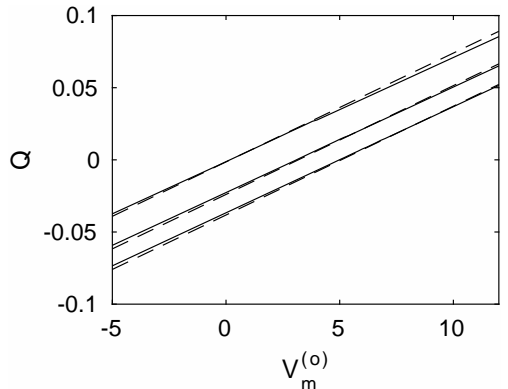

(b)

FIG. 4. (a) The true relationship between $V_{m}$ and $V_{m}^{(o)}$ (solid line), and the approximation given by Eq. (3.47) (broken line). (b) The true relationship between $Q$ and $V_{m}^{(o)}$ (solid line), and the approximation given by Eq. (3.52) (broken line). In both figures the top pair of lines were generated with $f=-0.06$, the middle pair of lines with $f=-1.5$, and the lower pair with $f=-4$. All variables plotted in this figure are dimensionless variables.

and that

$$
-Q=-\frac{\varepsilon_{\mathrm{m}}}{\varepsilon_{\mathrm{b}}} \frac{\partial \phi^{(M)}}{\partial X}=\int_{-\infty}^{-a} N_{e, 0}^{(D)}+K_{e, 0}^{(D)}+2 C_{e, 0}^{(D)}-A_{e, 0}^{(D)} \mathrm{d} X .
$$

Using Eqs. (3.36), (3.37), (3.47) and (3.49) we see that the approximate solution for the potential in the cell membrane allows us to approximate the charge by

$$
\begin{aligned}
Q & =C_{m} V_{m} \\
& =C_{m}\left(V_{m}^{(o)}+2 \log \left(\tanh \left(\frac{1}{2} \sinh ^{-1}\left(\frac{4}{-f} \sqrt{\frac{\varepsilon_{\mathrm{m}}\left(\bar{N}_{i}+\bar{K}_{i}\right)}{2 \varepsilon_{\mathrm{b}}}}\right)\right)\right)\right) .
\end{aligned}
$$

where the dimensionless capacitance $C_{m}$ is given by

$$
C_{m}=\frac{\varepsilon_{\mathrm{m}}}{2 a \varepsilon_{\mathrm{b}}} .
$$

In Figure 4(b) we compare the value of $Q$ calculated by solving the full system of equations (given Eqs. (3.21), (3.26) and (3.31), subject to the boundary conditions given by Eqs. (3.32)-(3.35), represented by the solid lines) with the approximation given by Eq. (3.52) (represented by the broken lines). The top pair of lines were generated using $f=-0.06$, the middle pair with $f=-1.5$, and the lower pair with $f=-4$. We see in Figure 4(b) that Eq. (3.51) is an excellent approximation to the full system of equations for all sets of parameters considered.

For consistency with the nondimensionalisation performed in Section 2.2 we write the dimensional capacitance $C_{m}^{*}$ as

$$
\begin{aligned}
C_{m}^{*} & =\bar{C}_{m} C_{m}, \\
\text { where } \quad \bar{C}_{m} & =\frac{\bar{\varepsilon}}{L \delta}
\end{aligned}
$$


The parameters given in Section 2.2 give $\bar{C}_{m}^{*} \approx 4.2 \times 10^{-3} \mathrm{~F} \mathrm{~m}^{-2}$, which is of the same order as the values reported elsewhere; see, for example, Beeler and Reuter (1977); Ebihara and Johnson (1980); Luo and Rudy (1994).

It is worth commenting upon the linearity of the capacitance relationships given by Eqs. (3.51) and (3.52), as this is an assumption made when deriving the bidomain equations. In Eq. (3.51) a capacitance relation is given for the quantity $V_{m}$ which, on appealing to the definition given by Eq. (3.45), we see is the true definition of the transmembrane potential. In this case, Eq. (3.51) predicts that the charge is proportional to $V_{m}$. In contrast, the quantity $V_{m}^{(o)}$ represents the transmembrane potential between the outer solutions for both intracellular and extracellular spaces, and neglects the Debye layers. In this case Eq. (3.52) predicts a linear relationship between $Q$ and $V_{m}^{(o)}$ with non-zero offset, rather than a proportionality relationship. However, when deriving the bidomain equations the capacitance relationship is differentiated with respect to time to give the capacitive current. Differentiation of Eqs. (3.51) and (3.52) with respect to time yields

$$
\frac{\partial Q}{\partial t}=C_{m} \frac{\partial V_{m}}{\partial t}, \quad \frac{\partial Q}{\partial t}=C_{m} \frac{\partial V_{m}^{(o)}}{\partial t},
$$

and so the extra constant that appears in Eq. (3.52), which arises due to the constant charge on the inner leaflet of the membrane adjacent to intracellular space, does not affect the capacitive current.

\subsection{Matching the outer solutions with the Debye layer solutions}

We have solutions for the leading order potential in the Debye layers, given by Eqs. (3.39) and (3.43) that: (i) satisfy the conditions at the cell membrane given by Eqs. (3.32)-(3.35); and (ii) match the leading order outer solution for the potentials given by Eqs. (3.8) and (3.13). We also have leading order solutions for the ion concentrations, given by Eqs. (3.22)-(3.25) in intracellular space and by Eqs. (3.27)(3.30) in extracellular space, that match the leading order outer solution for these ion concentrations. The one task remaining is to satisfy the flux boundary conditions on the leading order ion concentrations at the membrane, given by Eqs. (2.37)-(2.40) and Eqs. (2.43)-(2.46). This may be done using a similar method to that described by Richardson (2009), and so we give only an overview of this matching process and refer the interested reader to Richardson (2009) for more details.

Using the expansion given by Eq. (3.16), the first order term in Eq. (2.26) when using the rescaling given by Eq. (3.14) is

$$
\frac{\partial N_{i, 0}^{(D)}}{\partial t}+\frac{\partial \mathscr{F}_{N}^{(D)}}{\partial X}=0
$$

where the $\mathscr{O}(1)$ flux of sodium ions in the intracellular Debye layer is given by

$$
\mathscr{F}_{N}^{(D)}=\frac{\partial N_{i, 1}^{(D)}}{\partial X}+N_{i, 1}^{(D)} \frac{\partial \phi_{i, 0}^{(D)}}{\partial X}+N_{i, 0}^{(D)} \frac{\partial \phi_{i, 1}^{(D)}}{\partial X} .
$$

Integrating Eq. (3.53) across the Debye layer and applying the boundary condition Eq. (2.37) gives

$$
\frac{\partial}{\partial t} \int_{a}^{\infty} N_{i, 0}^{(D)} \mathrm{d} X=g_{N}-\lim _{X \rightarrow \infty} \mathscr{F}_{N}^{(D)}
$$


Similarly,

$$
\begin{aligned}
\frac{\partial}{\partial t} \int_{a}^{\infty} K_{i, 0}^{(D)} \mathrm{d} X & =g_{K}-\lim _{X \rightarrow \infty} \mathscr{F}_{K}^{(D)}, \\
\frac{\partial}{\partial t} \int_{a}^{\infty} C_{i, 0}^{(D)} \mathrm{d} X & =\frac{1}{2} g_{C}-\lim _{X \rightarrow \infty} \mathscr{F}_{C}^{(D)}, \\
\frac{\partial}{\partial t} \int_{a}^{\infty} A_{i, 0}^{(D)} \mathrm{d} X & =-\lim _{X \rightarrow \infty} \mathscr{F}_{A}^{(D)} .
\end{aligned}
$$

where $\mathscr{F}_{K}^{(D)}, \mathscr{F}_{C}^{(D)}, \mathscr{F}_{A}^{(D)}$ are the $\mathscr{O}(1)$ fluxes of potassium, calcium and other ions in the Debye layer. We may then use Eqs. (3.49) and (3.54)-(3.57) to deduce that

$$
\frac{\partial Q}{\partial t}=g_{N}+g_{K}+g_{C}-\lim _{X \rightarrow \infty}\left(\mathscr{F}_{N}^{(D)}+\mathscr{F}_{K}^{(D)}+2 \mathscr{F}_{C}^{(D)}-\mathscr{F}_{A}^{(D)}\right) .
$$

Richardson (2009) matched the leading order outer solutions to the leading order Debye layer solutions in both the intracellular and extracellular spaces by identifying an intermediate layer through which the potential, ion concentrations and total flux of ions is constant to leading order (although the fluxes of individual ions are not constant). The model used in the current study is slightly different to that used in Richardson (2009) as: (i) the permittivity is allowed to vary within the intracellular and extracellular spaces; and (ii) ions are modelled that do not have a valency with unit modulus. A very similar analysis can be carried out to deduce the existence of a very similar intermediate layer by using the same scaling as in Richardson (2009):

$$
x=\delta a+\delta^{1 / 2} w .
$$

Under this scaling, the leading order solution is independent of the permittivity, and so spatial variations in the permittivity have no effect. Further, it can be shown that, to leading order, the quantities $\phi, N_{i}, K_{i}$, $C_{i}, A_{i}$ and

$$
\mathscr{F}_{N}^{(\mathrm{int})}+\mathscr{F}_{K}^{(\mathrm{int})}+2 \mathscr{F}_{C}^{(\mathrm{int})}-\mathscr{F}_{A}^{(\mathrm{int})}
$$

are all constant, where $\mathscr{F}_{N}^{\text {(int) }}, \mathscr{F}_{K}^{\text {(int) }}, \mathscr{F}_{C}^{\text {(int) }}, \mathscr{F}_{A}^{\text {(int) }}$ are the $\mathscr{O}(1)$ fluxes of sodium, potassium, calcium and other ions in the intermediate layer. The intermediate layer then allows us to satisfy Eqs. (3.54)(3.57). Noting that the leading order fluxes of ions out of the intermediate layer as $X \rightarrow \infty$ will be balanced by the leading order intracellular current density in the outer region we can use Eqs. (3.9) and (3.58) to match the outer solution with the intermediate layer at leading order by demanding that

$$
\frac{\partial Q}{\partial t}=g_{N}+g_{K}+g_{C}-\sigma_{i} \frac{\partial \phi_{i, 0}^{(o)}}{\partial n}
$$

A similar argument may be used in the extracellular space, giving

$$
\frac{\partial Q}{\partial t}=g_{N}+g_{K}+g_{C}-\sigma_{e} \frac{\partial \phi_{e, 0}^{(o)}}{\partial n}
$$

Combining Eqs. (3.59) and (3.60) we see that, across the cell membrane,

$$
\sigma_{i} \frac{\partial \phi_{i, 0}^{(o)}}{\partial n}=\sigma_{e} \frac{\partial \phi_{e, 0}^{(o)}}{\partial n} .
$$




\subsection{The microscale equations}

We have derived a system of equations for the leading order outer solutions for the potential, which we now adapt so that they are in identical form to those used in other studies (Bruce et al., 2014; Hand and Griffith, 2010, 2011; Keener and Sneyd, 1998; Keener and Panfilov, 1996; Neu and Krassowska, 1993; Richardson and Chapman, 2011). Writing

$$
\tilde{\phi}_{i, 0}^{(o)}=\phi_{i, 0}^{(o)}+2 \log \left(\tanh \left(\frac{1}{2} \sinh ^{-1}\left(\frac{4}{-f} \sqrt{\frac{\varepsilon_{\mathrm{m}}\left(\bar{N}_{i}+\bar{K}_{i}\right)}{2 \varepsilon_{\mathrm{b}}}}\right)\right)\right)
$$

we see that, using Eq. (3.40) and noting that the logarithmic term in Eq. (3.62) is constant, the partial differential equations satisfied by $\phi_{i, 0}^{(o)}, \phi_{e}^{(o)}$, Eqs. (3.8) and (3.13), become:

$$
\begin{array}{ll}
\nabla^{2} \tilde{\phi}_{i, 0}^{(o)}=0, & \mathbf{x} \in \Omega_{i}, \\
\nabla^{2} \phi_{e, 0}^{(o)}=0, & \mathbf{x} \in \Omega_{e} .
\end{array}
$$

Using Eq. (3.51), the boundary conditions Eqs. (3.59) and (3.61) become

$$
\begin{aligned}
C_{m} \frac{\partial V_{m}}{\partial t} & =g_{N}+g_{K}+g_{C}-\sigma_{i} \frac{\partial \tilde{\phi}_{i, 0}^{(o)}}{\partial n}, & & \mathbf{x} \in \Omega_{i} \cap \Omega_{e}, \\
\sigma_{i} \frac{\partial \tilde{\phi}_{i, 0}^{(o)}}{\partial n} & =\sigma_{e} \frac{\partial \phi_{e, 0}^{(o)}}{\partial n}, & & \mathbf{x} \in \Omega_{i} \cap \Omega_{e},
\end{aligned}
$$

where, from Eq. (3.46),

$$
V_{m}=\tilde{\phi}_{i, 0}^{(o)}-\phi_{e, 0}^{(o)}
$$

These equations and boundary conditions differ only to those used in other studies (Bruce et al., 2014; Hand and Griffith, 2010, 2011; Keener and Sneyd, 1998; Keener and Panfilov, 1996; Neu and Krassowska, 1993; Richardson and Chapman, 2011) through the re-definition of the intracellular potential; the definition of $\tilde{\phi}_{i, 0}^{(o)}$ given by Eq. (3.62) is the intracellular potential in the Debye layer adjacent to the cell membrane rather than the outer solution for the intracellular potential.

\section{The macroscale equations}

Having derived our microscale model, we now utilise the homogenisation technique for an almost periodic microstructure described by Richardson and Chapman (2011) to derive the tissue level bidomain equations.

\subsection{The bidomain equations}

We define $\mathbf{x}$ to be the coordinates of a point in cardiac tissue, and assume that a transformation $\mathbf{B}(\mathbf{x}, t)$ exists such that the microstructure is periodic in the coordinate system $\mathbf{x}^{\prime}$, where

$$
\mathbf{x}^{\prime}=\mathbf{B}(\mathbf{x}, t)
$$


and we define the matrix $F$ by

$$
F_{i j}=\frac{\partial B_{j}}{\partial x_{i}}
$$

We acknowledge that this is a slight abuse of notation, as $F$ has previously been used to denote Faraday's constant. However, as $F$ is natural notation to use in the context above, and Faraday's constant will not be required for the remainder of this paper, this should not cause confusion. We note that $\mathbf{x}^{\prime}$ is a coordinate system in which the microstructure is periodic, and is not a coordinate system that represents the undeformed cardiac tissue before the onset of a heartbeat.

The purpose of the transformation $\mathbf{B}$ defined above is to transform the tissue microstructure in the $\mathbf{x}$ coordinate system so that it consists of identical repeating cardiac cells in the $\mathbf{x}^{\prime}$ coordinate system. We define $\mathbf{x}$ to be the macroscale coordinate, and assume that variations in the orientation of the microstructure occur over a lengthscale $L$ that is much longer than the length of an individual cardiac cell, $L_{\text {micro }}$. As a consequence we have

$$
\varepsilon=\frac{L_{\text {micro }}}{L} \ll 1
$$

We again note a slight abuse of notation, as $\varepsilon$ has been used to denote a permittivity earlier; this should not cause confusion as the permittivity is not under consideration in this section. Defining the microscale coordinate $\mathbf{y}^{\prime}$ by

$$
\mathbf{y}^{\prime}=\frac{\mathbf{x}^{\prime}}{\varepsilon}=\frac{\mathbf{B}(\mathbf{x}, t)}{\varepsilon}
$$

we see that components of $\mathbf{y}^{\prime}$ are $\mathscr{O}(1)$ over a single cardiac cell.

We now rescale some variables so that all current densities and potentials have magnitude that usually lie between 0 and 1 . Remembering the scaling for the current density used in Section 2.2 we rescale as follows, where carats denote scaled variables:

$$
\begin{aligned}
\hat{J} & =\frac{1}{0.233}\left(g_{N}+g_{K}+g_{C}\right), \\
\hat{\phi}_{i, 0}^{(o)} & =\frac{\tilde{\phi}_{i, 0}^{(o)}+3}{4} \\
\hat{\phi}_{e, 0}^{(o)} & =\frac{\phi_{e, 0}^{(o)}+3}{4} \\
\hat{V}_{m} & =\frac{V_{m}+3}{4} .
\end{aligned}
$$

We also rescale time so that

$$
\hat{t}=\frac{0.233 t}{4 C_{m}} .
$$


Eqs. (3.63)-(3.67) then become, on dropping carats:

$$
\begin{aligned}
\nabla^{2} \phi_{i, 0}^{(o)} & =0, & & \mathbf{x} \in \Omega_{i}, \\
\nabla^{2} \phi_{e, 0}^{(o)} & =0, & & \mathbf{x} \in \Omega_{e}, \\
\frac{\partial V_{m}}{\partial t} & =J-Z \frac{\partial \phi_{i, 0}^{(o)}}{\partial n}, & & \mathbf{x} \in \Omega_{i} \cap \Omega_{e}, \\
\sigma_{i} \frac{\partial \phi_{i, 0}^{(o)}}{\partial n} & =\sigma_{e} \frac{\partial \phi_{e, 0}^{(o)}}{\partial n}, & & \mathbf{x} \in \Omega_{i} \cap \Omega_{e}, \\
V_{m} & =\phi_{i, 0}^{(o)}-\phi_{e, 0}^{(o)}, & & \mathbf{x} \in \Omega_{i} \cap \Omega_{e},
\end{aligned}
$$

where $J=\mathscr{O}(1)$ and

$$
Z=\frac{4 \sigma_{i}}{0.233}
$$

Noting that myocytes are typically around $10^{-4} \mathrm{~m}$ long, and using the value of $L$ given in Section 2.2, we have

$$
\varepsilon=\frac{L_{m}}{L} \approx 0.1
$$

Using the definition of $\sigma_{i}$ given by Eq. (3.10), and the parameters given in Section 2.2, we obtain $Z=$ $\mathscr{O}(1 / \varepsilon)$, and so Eqs. (4.1)-(4.5) are precisely the distinguished limit of the microscale model investigated by Richardson and Chapman (2011), allowing us to obtain the tissue level bidomain equations

$$
\begin{aligned}
\nabla \cdot\left(\Sigma^{(i)} \nabla \Phi_{i}\right) & =\chi\left(J+C_{m}\left(\frac{\partial V}{\partial t}+(\mathbf{U} \cdot \nabla) V\right)\right) \\
\nabla \cdot\left(\Sigma^{(e)} \nabla \Phi_{e}\right) & =-\chi\left(J+C_{m}\left(\frac{\partial V}{\partial t}+(\mathbf{U} \cdot \nabla) V\right)\right)
\end{aligned}
$$

where $\Phi_{i}$ is the tissue level intracellular potential (which is actually representative of the intracellular potential adjacent to the cell membrane), $\Phi_{e}$ is the tissue level extracellular potential,

$$
V=\Phi_{i}-\Phi_{e}
$$

$\chi$ is the ratio of the dimensionless myocyte surface area to the dimensionless volume of the unit that we homogenise over, $\mathbf{U}$ is the velocity of the tissue, and $\Sigma^{(i)}, \Sigma^{(e)}$ are the tissue level intracellular and extracellular conductivity tensors that arise from the homogenisation procedure.

The conductivity tensors may be written in terms of the solution of differential equations that would normally be termed "cell problems". However, as the use of the word "cell" in this context could cause confusion with cardiac cells, we abandon this convention and will refer to these problems as being defined on the unit that we homogenise over. These problems may be specified in several equivalent forms, see Richardson and Chapman (2011) for a full discussion. For the purpose of interpreting the homogenised conductivity tensors we define the conductivity tensors as having entries given by, for 
$p, r=1,2,3:$

$$
\begin{aligned}
& \Sigma_{p r}^{(i)}=\frac{\Theta \sigma_{i}}{V^{\prime}} F_{p j}\left(\int_{\Omega_{i}^{\prime}} \delta_{j k}+\frac{\partial W_{k}^{(i)}}{\partial y_{j}^{\prime}} \mathrm{d} V^{\prime}\right) F_{k r}^{-1}, \\
& \Sigma_{p r}^{(e)}=\frac{\Theta \sigma_{e}}{V^{\prime}} F_{p j}\left(\int_{\Omega_{e}^{\prime}} \delta_{j k}+\frac{\partial W_{k}^{(e)}}{\partial y_{j}^{\prime}} \mathrm{d} V^{\prime}\right) F_{k r}^{-1},
\end{aligned}
$$

where $\Theta=\varepsilon Z$, and, here and throughout the remainder of this paper, we will use the summation convention. The vectors $\mathbf{W}^{(\mathbf{i})}, \mathbf{W}^{(\mathbf{e})}$ are periodic in $\mathbf{y}^{\prime}$ and satisfy, for $r=1,2,3$ :

$$
\begin{array}{rlrl}
F_{i j} F_{i k} \frac{\partial^{2} W_{r}^{(i)}}{\partial y_{j}^{\prime} \partial y_{k}^{\prime}} & =0, & & \mathbf{y}^{\prime} \in \Omega_{i}^{\prime}, \\
F_{i j} F_{i k} n_{j}^{\prime} \frac{\partial W_{r}^{(i)}}{\partial y_{k}^{\prime}} & =F_{p q} F_{p r} n_{q}^{\prime}, & \mathbf{y}^{\prime} \in \partial \Omega^{\prime}, \\
F_{i j} F_{i k} \frac{\partial^{2} W_{r}^{(e)}}{\partial y_{j}^{\prime} \partial y_{k}^{\prime}}=0, & \mathbf{y}^{\prime} \in \Omega_{e}^{\prime}, \\
F_{i j} F_{i k} n_{j}^{\prime} \frac{\partial W_{r}^{(e)}}{\partial y_{k}^{\prime}}=F_{p q} F_{p r} n_{q}^{\prime}, & \mathbf{y}^{\prime} \in \partial \Omega^{\prime},
\end{array}
$$

where, in the $\mathbf{y}^{\prime}$ coordinate system, $\mathbf{n}^{\prime}$ is the unit normal pointing from intracellular space into extracellular space, $\Omega_{i}^{\prime}$ is the intracellular region, $\Omega_{e}^{\prime}$ is the extracellular region, and $\partial \Omega^{\prime}$ is the cell membrane (now assumed to have zero thickness). We prove in Appendix B that the conductivity tensors defined above are both symmetric and positive definite. This ensures the existence of an orthonormal change of coordinates, with respect to which a conductivity tensor is diagonal with positive entries on the diagonal.

A common assumption is that the convection term in Eqs. (4.6) and (4.7) may be neglected. An evaluation of this assumption would require us to calculate the tissue velocity $\mathbf{U}$ which would, in turn, require a description of the forces generated; see, for example Hunter et al. (1998); Niederer et al. (2006). This would add significant complexity into the discussion, and we postpone a discussion of the effects of neglecting this term to future work.

\subsection{Analytic predictions on entries of the conductivity tensors}

We now present some observations on the entries of the conductivity tensors, and some approximations that are relevant to cardiac tissue. Further observations arising from calculating the numerical solution of Eqs. (4.11)-(4.14) are given in Section 4.3.

We use the singular value decomposition theorem (see, for example, Trefethen and Bau (1997)) to write $F$ as the product of linear transformations. This theorem allows us to factorise any square matrix $F$ with real entries as

$$
F=Q D P,
$$

where $P, Q, R$ are square matrices of the same size as $F$ and

$$
P^{\top} P=\mathscr{I}, \quad Q^{\top} Q=\mathscr{I}, \quad D \text { is diagonal with non-negative entries, }
$$


where $\mathscr{I}$ is the identity matrix. Furthermore, the values that appear on the diagonal of $D$ are uniquely defined. We may then interpret $F$ as a combination of a rotation and a reflection (represented by $P$ ), followed by a stretch along each coordinate axis (represented by $D$ ), followed by a further combination of a rotation and reflection (represented by $Q$ ). We now interpret the conductivity tensors in terms of the matrices $P, Q, R$.

4.2.1 The effect of $Q$. The differential equations and boundary conditions on the unit that we homogenise over, given by Eqs. (4.11)-(4.14), depend on $F$ only through terms of the form $F_{i j} F_{i k}$, which is the $j, k$ entry of $F^{\top} F$. It is therefore $F^{\top} F$, rather than $F$, that is of interest when solving Eqs. (4.11)(4.14). The singular value decomposition of $F$ given by Eq. (4.15), together with the properties of $Q$ and $D$ given by Eq. (4.16), allow us to write $F^{\top} F=P^{\top} D^{2} P$, which is idependent of $Q$, and so the solution of Eqs. (4.11)-(4.14) is also independent of $Q$. Suppose $\Sigma^{(i, D P)}, \Sigma^{(e, D P)}$ are the conductivity tensors arising when $F=D P$. We then have, on using Eqs. (4.9) and (4.10),

$$
\Sigma_{p r}^{(i)}=Q \Sigma^{(i, P D)} Q^{\top}, \quad \Sigma_{p r}^{(e)}=Q \Sigma^{(e, P D)} Q^{\top} .
$$

This is expected; it is a statement that if tissue is rotated after stretches have been carried out, with the rotation matrix represented by $Q$, then this rotation simply aligns the conductivity tensor with a coordinate system that has been rotated using the same matrix $Q$.

4.2.2 The special case that $F=D$. Suppose the repeating unit that we homogenise over is symmetric in the planes $y_{1}^{\prime}=y_{1}^{(0)^{\prime}}, y_{2}^{\prime}=y_{2}^{(0)^{\prime}}$ and $y_{3}^{\prime}=y_{3}^{(0)^{\prime}}$. We will now demonstrate that if $F$ is a diagonal matrix, then these symmetries allow us to deduce that the conductivity tensors defined by Eq. (4.9) and (4.10) are diagonal. If $F$ is given by

$$
F=\left(\begin{array}{ccc}
D_{11} & 0 & 0 \\
0 & D_{22} & 0 \\
0 & 0 & D_{33}
\end{array}\right)
$$

then the unit problem given by Eq. (4.11) for $W_{1}^{(i)}$ becomes, for $\mathbf{y}^{\prime} \in \Omega_{i}^{\prime}$ :

$$
D_{11}^{2} \frac{\partial^{2} W_{1}^{(i)}}{\partial y_{1}^{\prime 2}}+D_{22}^{2} \frac{\partial^{2} W_{1}^{(i)}}{\partial y_{2}^{\prime 2}}+D_{33}^{2} \frac{\partial^{2} W_{1}^{(i)}}{\partial y_{3}^{\prime 2}}=0
$$

and the boundary condition given by Eq. (4.12) becomes, for $\mathbf{y}^{\prime} \in \partial \Omega^{\prime}$ :

$$
D_{11}^{2} \frac{\partial W_{1}^{(i)}}{\partial y_{1}^{\prime}} n_{1}^{\prime}+D_{22}^{2} \frac{\partial W_{1}^{(i)}}{\partial y_{2}^{\prime}} n_{2}^{\prime}+D_{33}^{2} \frac{\partial W_{1}^{(i)}}{\partial y_{3}^{\prime}} n_{3}^{\prime}=-D_{11}^{2} \frac{\partial W_{1}^{(i)}}{\partial y_{1}^{\prime}} n_{1}^{\prime} .
$$

Suppose a solution $W_{1}^{(i)}$ is found that satisfies both the differential equation given by Eq. (4.17) and the boundary conditions given by Eq. (4.18) in the region $y_{2}^{\prime}>y_{2}^{(0)^{\prime}}$. We may then extend the definition of $W_{1}^{(i)}$ by setting, for $y_{2}^{\prime}<y_{2}^{(0)^{\prime}}$ :

$$
W_{1}^{(i)}\left(y_{1}^{\prime}, y_{2}^{\prime}-y_{2}^{(0)^{\prime}}, y_{3}^{\prime}\right)=W_{1}^{(i)}\left(y_{1}^{\prime},-\left(y_{2}^{\prime}-y_{2}^{(0)^{\prime}}\right), y_{3}^{\prime}\right) .
$$


We see that this extended definition of $W_{1}^{(i)}$ satisfies the differential equation given by Eq. (4.17) and the boundary conditions given by Eq. (4.18) at all points in the domain under consideration. The solution $W_{1}^{(i)}$ is then an even function of $y_{2}^{\prime}$ about the plane $y_{2}^{\prime}=y_{2}^{(0)^{\prime}}$, and so the partial derivative $\partial W_{1}^{(i)} / \partial y_{2}^{\prime}$ is an odd function of $y_{2}^{\prime}$ about this plane. If we define $B$ to be the matrix with entries given by

$$
B_{j k}=\int_{\Omega_{i}^{\prime}} \delta_{j k}+\frac{\partial W_{k}^{(i)}}{\partial y_{j}^{\prime}} \mathrm{d} V^{\prime}, \quad j, k=1,2,3
$$

then using Eq. (4.9), and recalling the symmetry of the region of integration about $y_{2}^{\prime}=y_{2}^{(0)^{\prime}}$, we may deduce that $B_{12}=0$. Other off-diagonal entries of $B$ can be shown to be zero using similar arguments. Noting that $D A D^{-1}=A$ for all diagonal matrices $A, D$ (provided $D^{-1}$ exists), Eq. (4.9) allows us to deduce that the intracellular conductivity tensor is diagonal. A similar argument may be used to deduce that the extracellular conductivity tensor is also a diagonal matrix.

4.2.3 The special case that $P D=D P$ and $P$ is a rotation matrix. Let $P$ be a $3 \times 3$ orthogonal matrix representing a non-trivial rotation, and $D$ be a $3 \times 3$ diagonal matrix $D$. In Appendix $\mathrm{C}$ we prove that $P$ and $D$ commute provided that either: (i) the axis of the rotation lies along one of the coordinate axes, and the entries of $D$ that represent the stretch along the other two axes are equal; or (ii) $D$ is a multiple of the identity matrix.

Assuming that the property that $P D=D P$ holds we obtain

$$
F=Q D P=\hat{Q} D
$$

where $\hat{Q}=Q P$ is an orthonormal matrix. The discussion in Section 4.2.1 then allows us to deduce that, if $\Sigma^{(i, D)}, \Sigma^{(e, D)}$ are the conductivity tensors arising when $F=D$, we then have, using Eqs. (4.9)-(4.10):

$$
\Sigma^{(i)}=(Q P) \Sigma^{(i, D)}(Q P)^{\top}, \quad \Sigma^{(e)}=(Q P) \Sigma^{(e, D)}(Q P)^{\top} .
$$

In this special case when the matrices $P$ and $D$ commute we may calculate the conductivity tensors by first compute the conductivity tensors arising from the stretches, and then applying the composite rotation and reflection described by the matrix $Q P$.

4.2.4 An approximation relevant to cardiac tissue. Models of cardiac tissue deformation are often singular outside the region $0.85<\lambda<1.2$, where $\lambda$ is the stretch ratio in the direction of the cardiac fibre of the deformed tissue relative to the undeformed tissue; see, for example, Hunter et al. (1998). If we assume that this stretch is representative of the maximum stretch in all directions, and is also representative of the stretch in all directions between the deformed tissue and a periodic microstructure, the matrix $D$ that appears in the singular value decomposition of $F$ given by Eq. (4.15) may be written

$$
D=\mathscr{I}+\delta\left(\begin{array}{ccc}
\hat{D}_{11} & 0 & 0 \\
0 & \hat{D}_{22} & 0 \\
0 & 0 & \hat{D}_{33}
\end{array}\right)
$$

where $\delta=\mathscr{O}(0.1)$, and $\hat{D}_{11}, \hat{D}_{22}, \hat{D}_{33}=\mathscr{O}(1)$. We note another slight abuse of notation; $\delta$ was previously used to denote a small parameter when deriving the microscale model in Sections 2 and 3. However, this new use of $\delta$ is confined to this short subsection, and we feel the discussion in this section 
will be clearer if we use a symbol that is commonly used for a small parameter. Using Eq. (4.19) we have, for any matrix $P$ with entries that are all $\mathscr{O}(1)$ :

$$
D P=P D+B,
$$

where all entries of the matrix $B$ are $\mathscr{O}(\delta)$. Using the singular value decomposition of $F$, and noting that all entries of $P$ and $P$ are $\mathscr{O}(1)$, we obtain

$$
\begin{aligned}
F & =Q D P \\
& =Q P D+Q B \\
& =Q P+C,
\end{aligned}
$$

where all entries of the matrix $C$ are also $\mathscr{O}(\delta)$. We now expand $W_{r}^{(i)}, r=1,2,3$, as a regular asymptotic expansion in powers of $\delta$ :

$$
W_{r}^{(i)}=W_{r, 0}^{(i)}+\delta W_{r, 1}^{(i)}+\ldots,
$$

and see, on using Eqs. (4.11) and (4.12) that the leading order term satisfies

$$
\begin{aligned}
\frac{\partial^{2} W_{r, 0}^{(i)}}{\partial y_{j}^{\prime} \partial y_{j}^{\prime}} & =0, \quad \mathbf{y}^{\prime} \in \Omega_{i}^{\prime}, \\
n_{k}^{\prime} \frac{\partial W_{r, 0}^{(i)}}{\partial y_{k}^{\prime}} & =n_{r}^{\prime}, \quad \mathbf{y}^{\prime} \in \partial \Omega^{\prime},
\end{aligned}
$$

and so the leading order term for $W_{r, 0}^{(i)}$ is given by the solution of Eqs. (4.11) and (4.12) with $F=\mathscr{I}$. Substituting the asymptotic expansion for $W_{r, 0}^{(i)}$, and the expression for $F$ given by Eq. (4.20), into Eq. (4.9) gives, on neglecting terms of $\mathscr{O}(\delta)$ :

$$
\Sigma^{(i)}=(Q P) \Sigma^{(i, \mathscr{I})}(Q P)^{\top}
$$

where $\Sigma^{(i, \mathscr{I})}$ is the intracellular conductivity tensor obtained when $F=\mathscr{I}$. Similarly, neglecting terms of $\mathscr{O}(\delta)$ allows us to approximate the extracellular conductivity tensor by

$$
\Sigma^{(e)}=(Q P) \Sigma^{(e, \mathscr{I})}(Q P)^{\top},
$$

where $\Sigma^{(e, \mathscr{I})}$ is the extracellular conductivity tensor obtained when $F=\mathscr{I}$.

The assumption given by Eq. (4.19) — that the stretch ratios in all directions are given by $1+\mathscr{O}(\delta)$ for $\delta \ll 1$ - allows us to verify the more general assumption used when modelling cardiac tissue using the bidomain equations: given principal values of the conductivity tensors, the conductivity tensors at a given point may be approximated by rotating the diagonal conductivity tensor to align with the microstructure, and that the diagonal entries are constant across the tissue. We will verify the validity of this assumption numerically in Section 4.3.

\subsection{Numerical predictions on entries of the conductivity tensors}

We now extend some of the ideas given in Section 4.2 by calculating a numerical solution of Eqs. (4.11)(4.14), the differential equations and boundary conditions on the unit we homogenise over, and then using Eqs. (4.9) and (4.10) to compute the conductivity tensors. In all calculations we set $\Theta=\sigma_{i}=$ $\sigma_{e}=1$ in Eqs. (4.9) and (4.10). 
4.3.1 The repeating unit that we homogenise over. Before we may calculate a numerical solution of Eqs. (4.11)-(4.14) we first need to generate a repeating unit to homogenise over. Myocytes are roughly of a long cylindrical shape, and we align the axis of the cylinder with the $y_{3}^{\prime}$-axis. The repeating periodic unit in $\mathbf{y}^{\prime}$ coordinates that we homogenise over occupies the region $-L_{1}<y_{1}^{\prime}<L_{1},-L_{2}<y_{2}^{\prime}<L_{2}$, $-0.5<y_{3}^{\prime}<0.5$. The unit is then constructed in three steps. As a first step we define the cell membrane to be a cylinder, with axis in the $y_{3}^{\prime}$ direction, with radius $r\left(y_{3}^{\prime}\right)$ given by

$$
r=R_{1}+\frac{1}{2}\left(R_{0}-R_{1}\right)\left(1+\tanh K\left(\left|y_{3}^{\prime}\right|-Z_{0}\right)\right)
$$

where $R_{0}<R_{1}$, and $R_{1}<L_{1}, L_{2}$. We define the intracellular region to be the interior of this cylinder, the extracellular region to be the exterior of this region, and use the parameters $R_{0}=0.1, R_{1}=0.11, K=$ $20, Z_{0}=0.45, L_{1}=0.111, L_{2}=0.111$. The second step is to incorporate connectivity between the intracellular region of adjacent repeating units in the $y_{1}^{\prime}$ direction. To this end, we first remove the regions where $\left(y_{2}^{\prime}\right)^{2}+\left(y_{3}^{\prime}\right)^{2}<r_{23}^{2}$ from the cell membrane, and add surfaces where $\left(y_{2}^{\prime}\right)^{2}+\left(y_{3}^{\prime}\right)^{2}=r_{23}^{2}$ that join the cell membrane to the boundary of the unit that we homogenise over. The third step is to add connectivity of the intracellular region of adjacent repeating units in the $y_{2}^{\prime}$ direction in a similar manner to that done in the second step for the $y_{1}^{\prime}$ direction. To do this we remove the regions where $\left(y_{1}^{\prime}\right)^{2}+\left(y_{3}^{\prime}\right)^{2}<r_{13}^{2}$, from the cell membrane, and add surfaces where $\left(y_{1}^{\prime}\right)^{2}+\left(y_{3}^{\prime}\right)^{2}=r_{13}^{2}$ that join the cell membrane to the boundary of the unit that we homogenise over. We use two different sets of parameters for $r_{23}$ and $r_{13}$ in our simulations. For the first set, we use $r_{23}=0.05, r_{13}=0.04$. For the second set we use values with a more marked difference and set $r_{23}=0.06, r_{13}=0.02$.

We give an exemplar plot the unit that we homogenise over in Figure 5. To illustrate this unit clearly we plot the unit using values of exaggerated values of $R_{0}, L_{1}, L_{2}, r_{23}, r_{13}$, taking $R_{1}=0.15, L_{1}=L_{2}=0.4$, $r_{23}=0.06, r_{13}=0.03$. We emphasise that these values are for clarity of illustration of the unit only. The values specified earlier result in a unit that we homogenise over that is more representative of the configuration of myocytes reported elsewhere (Guyton and Hall, 1996).

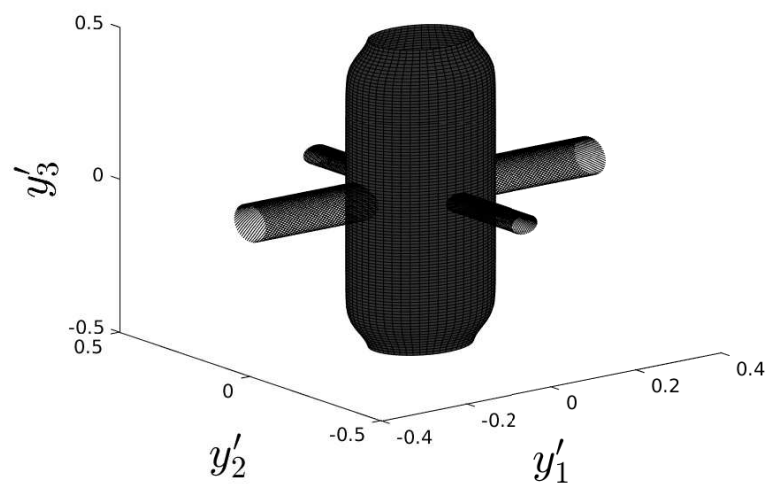

FIG. 5. The repeating unit that we homogenise over. Note that the parameters used in this figure are chosen for clarity; see text for more details on the parameters used in simulations. 
4.3.2 The special case that $F=D$. We noted in Section 4.2.2 that when $F=D$, representing stretches in the direction of the coordinate axes, both the intracellular and extracellular conductivity tensors are diagonal matrices. In Figure 6 we plot the diagonal entries of the intracellular and extracellular conductivity tensors when

$$
F=\left(\begin{array}{ccc}
\lambda_{1} & 0 & 0 \\
0 & \lambda_{1}^{-1 / 2} & 0 \\
0 & 0 & \lambda_{1}^{-1 / 2}
\end{array}\right)
$$

for $0.8<\lambda_{1}<1.2$, i.e. the range of stretches for cardiac tissue discussed in Section 4.2.4. We begin by using the first set of parameters given in Section 4.3.1 to generate the repeating unit that we homogenise over. The diagonal entries of $\Sigma^{(i)}$ and $\Sigma^{(e)}$ that are generated using this repeating unit are plotted Figures 6(a) and 6(b). We see that the entries vary very little over these values of $\lambda_{1}$, in agreement with the predictions in Section 4.2.4. We then consider the second set of parameters given in Section 4.3.1 to generate the repeating unit that we homogenise over, and plot the diagonal entries of $\Sigma^{(i)}$ and $\Sigma^{(e)}$ that are generated in Figures 6(c) and 6(d). There is slightly more dependence on $\lambda_{1}$ than for the first set of parameters, although the dependence is still modest.

4.3.3 Effect of shearing. We now investigate the effect of a transformation between a periodic microstructure and an almost periodic microstructure that is given by

$$
x_{1}=x_{1}^{\prime}, \quad x_{2}=x_{2}^{\prime}, \quad x_{3}=x_{3}^{\prime}+\gamma\left(x_{1}^{\prime}+x_{2}^{\prime}\right) .
$$

This represents a shear, and the matrix $F$ that represents this shear is given by

$$
F=\left(\begin{array}{ccc}
1 & 0 & -\gamma \\
0 & 1 & -\gamma \\
0 & 0 & 1
\end{array}\right)
$$

We note that this shear does not change the fibre direction, sheet direction or normal to these two directions, and recall that these directions are commonly assumed to determine the principal directions of the conductivity tensors.

We begin by using the first set of parameters in Section 4.3.1 to generate the repeating unit that we homogenise over, and generate $\Sigma^{(i)}$ and $\Sigma^{(e)}$ by solving Eqs. (4.9)-(4.14). As $\Sigma^{(i)}$ and $\Sigma^{(e)}$ are symmetric and positive definite we may calculate the principal values and principal directions. The principal directions are given by the eigenvectors of $\Sigma^{(i)}$ and $\Sigma^{(e)}$. We use spherical polar coordinates to represent the principal directions, where a point in space is defined by coordinates

$$
(r \cos \phi \sin \theta, r \sin \phi \sin \theta, r \cos \theta)
$$

where $r \geqslant 0,0 \leqslant \theta<\pi$ and $0 \leqslant \phi<2 \pi$. Using spherical polar coordinates, the coordinate axis $x_{1}$ is in the direction given by $\theta=\pi / 2, \phi=0, x_{2}$ is in the direction given by $\theta=\pi / 2, \phi=\pi / 2$, and $x_{3}$ is in the direction given by $\theta=0$, with $\phi$ undetermined.

We plot the eigenvalues of $\Sigma^{(i)}$ and $\Sigma^{(e)}$ as a function of $\gamma$ in Figures 7(a) and 7(b), and the values of $\theta$ for the principal directions in Figures 7(c) and 7(d). We see that these vary very little with $\gamma$, and that the value of $\theta$ is the expected value. We plot the values of $\phi$ for the principal directions in 


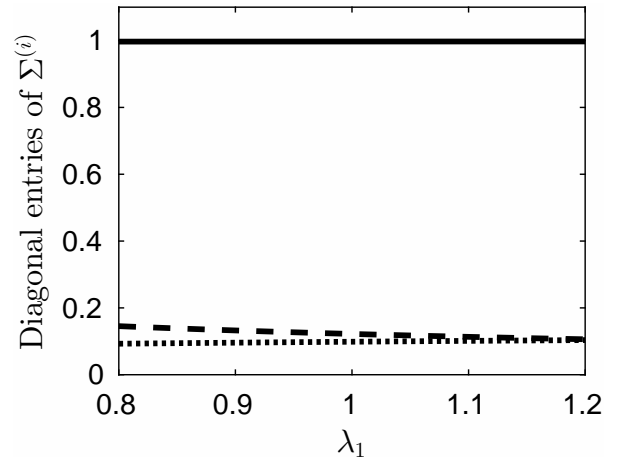

(a)

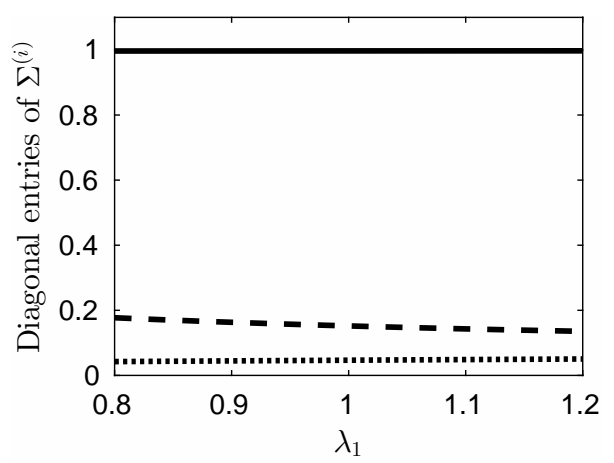

(c)

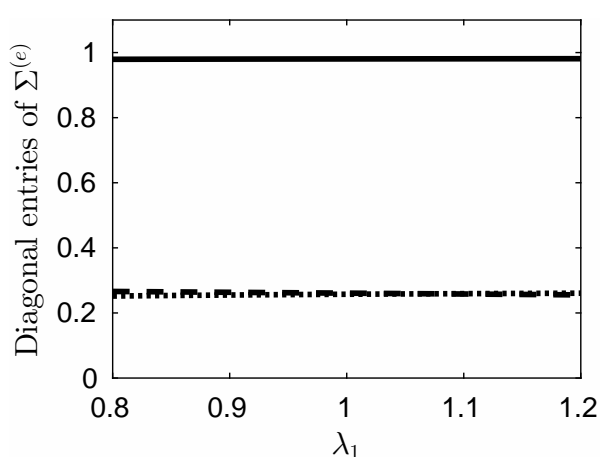

(b)

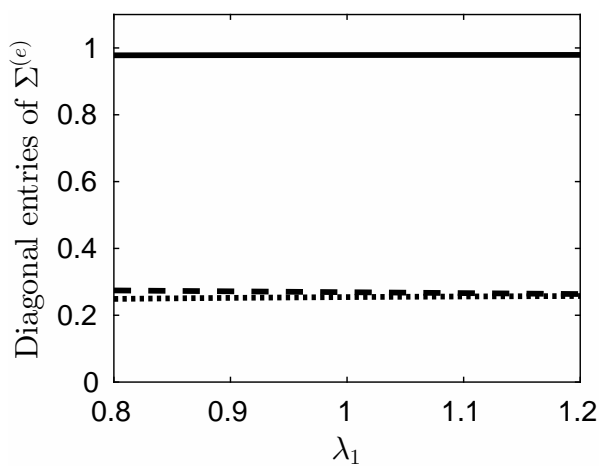

(d)

FIG. 6. The diagonal entries of the conductivity tensors as a function of the stretch $\lambda_{1}$; see text for more details on the definition of the stretch. (a) The entries of $\Sigma^{(i)}$, and (b) the diagonal entries of $\Sigma^{(e)}$ when using the first set of parameters given in Section 4.3 .1 to generate the repeating unit that we homogenise over. (c) and (d) show the diagonal entries of $\Sigma^{(i)}$ and $\Sigma^{(e)}$ when using the second set of parameters to generate the repeating unit that we homogenise over. In all figures the solid line represents the conductivity in the $x_{3}$ direction (i.e. the entry in row 3 and column 3 of the conductivity tensor), the broken line the conductivity in the $x_{1}$ direction, and the dotted line the conductivity in the $x_{2}$ direction.

Figures 7(e) and 7(f). In this figure we do not plot the values of $\phi$ when $\theta=0$, as under these conditions $\phi$ is not determined (or needed). We do see some variation in $\phi$ with $\gamma$, particularly for the extracellular conductivity tensor. However, we saw in Figures 7(a) and 7(b) that two of the conductivities are very similar, again especially for the extracellular conductivity tensor. Noting that the principal direction associated with the two similar conductivities has $\theta=\pi / 2$, under these circumstances variations in $\phi$ for these two conductivities will have very little effect on the conductivity in a given direction. As a consequence these variations in $\phi$ are likely to have only minimal effect on the solution of the tissue level bidomain equations.

We now repeat the calculation of $\Sigma^{(i)}$ and $\Sigma^{(e)}$ presented in Figure 7, using the second set of parameters described in Section 4.3.1 to generate the repeating unit that we homogenise over. We plot the eigenvalues of $\Sigma^{(i)}$ and $\Sigma^{(e)}$ as a function of $\gamma$ in Figures 8(a) and 8(b). In common with the calculations presented in Figures 7(a) and 7(b) we see that the dependence of the eigenvalues on $\gamma$ is very weak. In 


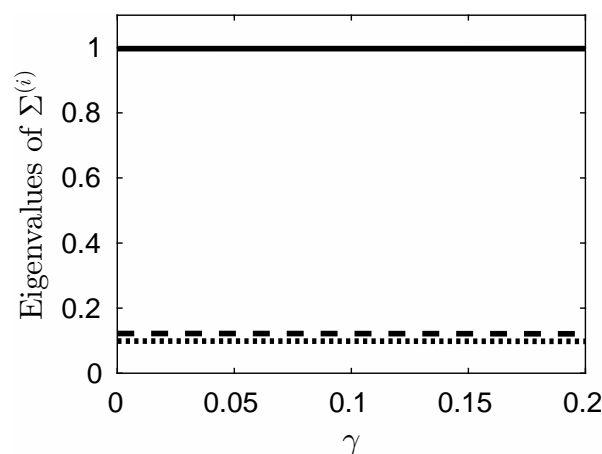

(a)

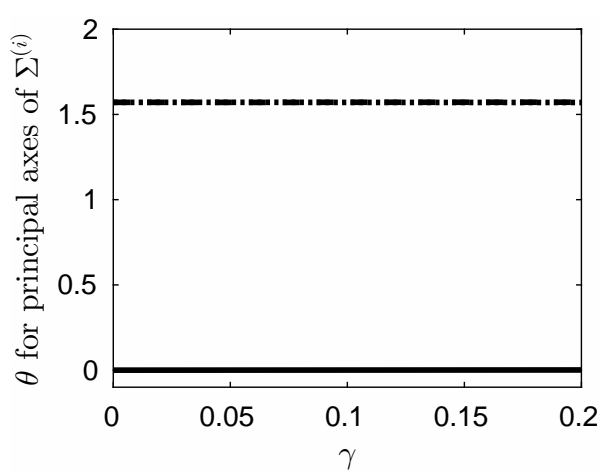

(c)

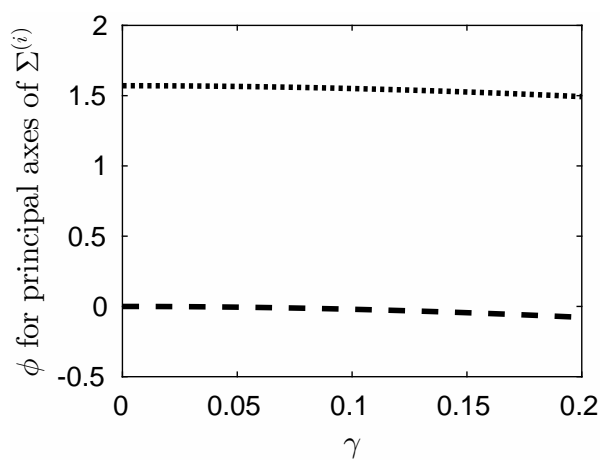

(e)

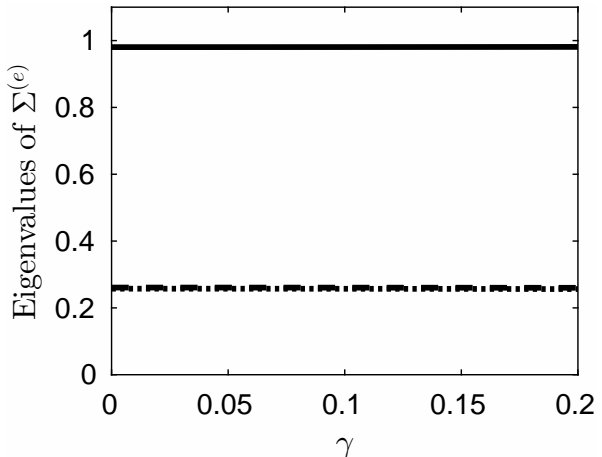

(b)

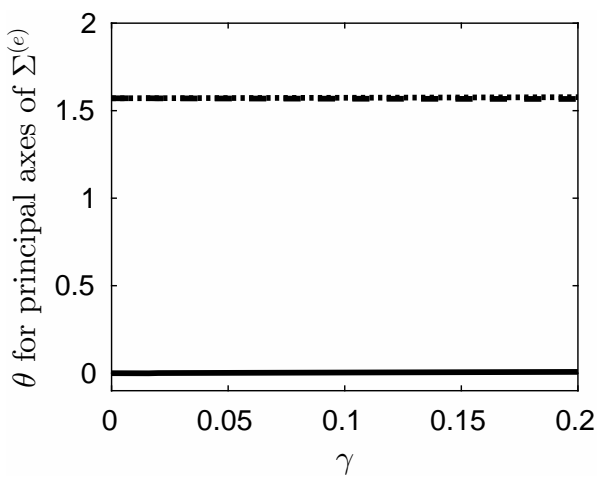

(d)

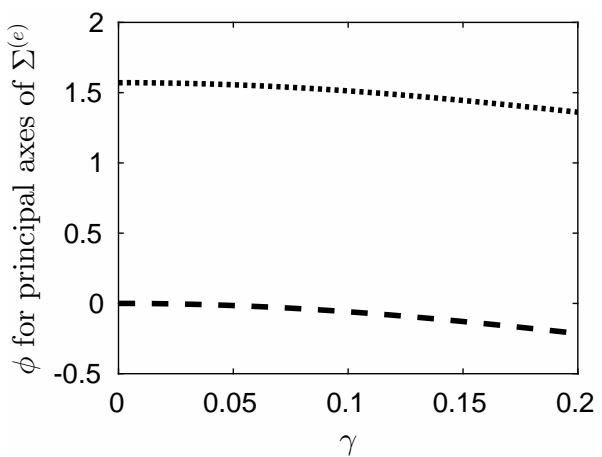

(f)

FIG. 7. (a), (b) the eigenvalues of $\Sigma^{(i)}$ and $\Sigma^{(e)}$; (c), (d) the $\theta$ coordinate in spherical polar coordinates of the eigenvectors of $\Sigma^{(i)}$ and $\Sigma^{(e)} ;$ (e), (f) the $\phi$ coordinate in spherical polar coordinates of the eigenvectors of $\Sigma^{(i)}$ and $\Sigma^{(e)}$. The first set of parameters in Section 4.3.1 was used to generate the repeating unit that we homogenise over. In all figures the solid line represents the principal component corresponding to the fibre direction, the broken line the sheet direction, and the dotted line the sheet normal direction. 
contrast to Figures 8(a) and 8(b), however, the eigenvalues are more widely separated. In Figures 8(c) and $8(\mathrm{~d})$ we again see that the values of $\theta$ calculated vary very little with $\gamma$, and take expected values. As the eigenvalues are now more separated than those in Figure 7 we see in Figures 8(e) and 8(f) that the values of $\phi$ vary far less with $\gamma$. As a consequence, the principal directions of both conductivity tensors are very close to being aligned with the axes of the $\mathbf{x}$ coordinate system, as would be expected when the microstructure orientation is unchanged. Given the weak dependence on $\gamma$, use of the conductivity tensors generated when $\gamma=0-$ i.e. the microstructure that has not been sheared - is an excellent approximation to the conductivity tensors that are calculated using other values of $\gamma$.

4.3.4 Larger stretches that are not along coordinate axes. In our final set of calculations we investigate stretches that are larger than those considered so far, and that are large enough that the approximations derived in Section 4.2.4 are not valid. Although these may not normally be seen when modelling cardiac tissue, they may be representative of a precordial thump (see, for example, Pellis and Kohl (2009)) where an attempt is made to restart the heart through a violent impact.

We use $F=D P$, where

$$
D=\left(\begin{array}{ccc}
\lambda_{1} & 0 & 0 \\
0 & \lambda_{1}^{-1 / 2} & 0 \\
0 & 0 & \lambda_{1}^{-1 / 2}
\end{array}\right), \quad P=\left(\begin{array}{ccc}
\cos \frac{1}{4} \pi & 0 & -\sin \frac{1}{4} \pi \\
0 & 1 & 0 \\
\sin \frac{1}{4} \pi & 0 & \cos \frac{1}{4} \pi
\end{array}\right)
$$

and so $F$ represents an anti-clockwise rotation through an angle $\pi / 4$ about the positive $x_{2}$ axis, followed by stretches along the original directions of the coordinate axes. Under the given rotation the $x_{1}$ axis is rotated to point in the direction $\theta=\pi / 4, \phi=0$, the $x_{2}$ axis in the direction $\theta=\pi / 2, \phi=\pi / 2$, and the $x_{3}$ axis in the direction $\theta=\pi / 4, \phi=\pi$.

We calculate $\Sigma^{(i)}$ and $\Sigma^{(e)}$ using the second set of parameters described in Section 4.3.1 to generate the repeating unit that we homogenise over. We plot the eigenvalues of $\Sigma^{(i)}$ and $\Sigma^{(e)}$ as a function of $\lambda_{1}$ in Figures 9(a) and 9(b), and see that these eigenvalues are more dependent on $\lambda_{1}$ than those in earlier simulations, especially the eigenvalue representing the conductivity in the sheet direction, perpendicular to the fibre direction. We also see, in Figures 9(c) and 9(d), that the values of $\theta$ also varies from the value that would occur if the matrices $P$ and $D$ commuted. Finally, for the choice of $P$ and $D$ used in these calculations, we see in Figures 9(e) and 9(f) that $\phi$ is largely unaffected by changes in $\lambda_{1}$. This may, of course, not be the case if we used a different rotation; the rotation described by the matrix $P$ does not change the value of $\phi$ when applied to a point in space.

The key point to note from the calculation presented in this section is that, for large stretches, both the principal conductivities of the conductivity tensors, and their principal directions, depend on the stretch. When $\lambda_{1}$ is close to the value 1 , the analysis in Section 4.2.4 allows us to write $F \approx Q D$ with $Q=P$. Furthermore, when using this approximation, the result from Section 4.2.1 allows us to deduce that the principal directions are then determined by the rotation matrix $P$, and we see the principal directions in Figure 9 when $\lambda_{1}=1$ are indeed those predicted earlier in this section. However, as $\lambda_{1}$ moves away from the value 1 , these values diverge from those predicted by assuming that the rotation and the stretch matrices commute. Under these circumstances it is therefore no longer valid to assume that the principal values of the conductivity tensor are uniform across tissue, and that the principal axes of these tensors may be aligned with the microstructure orientation. 


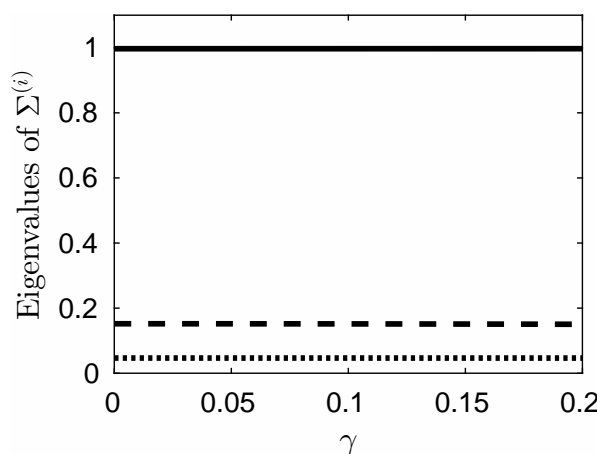

(a)

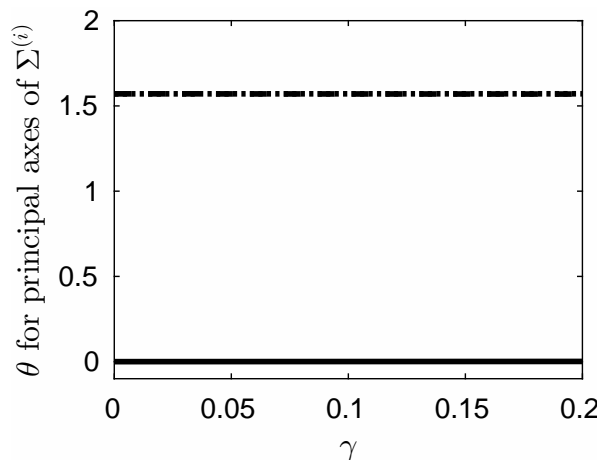

(c)

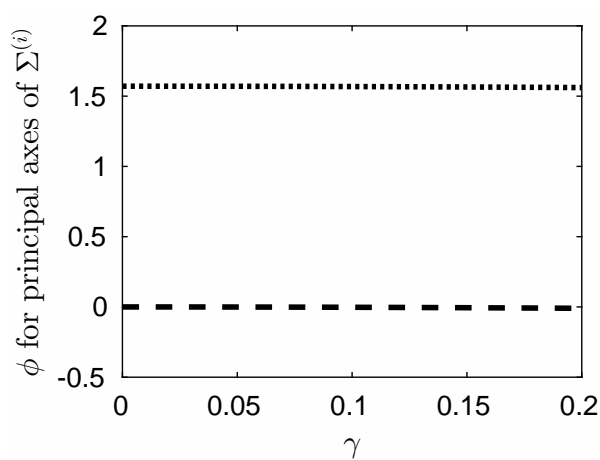

(e)

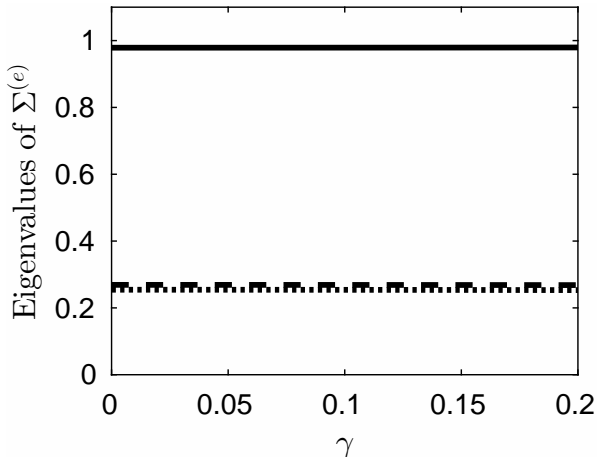

(b)

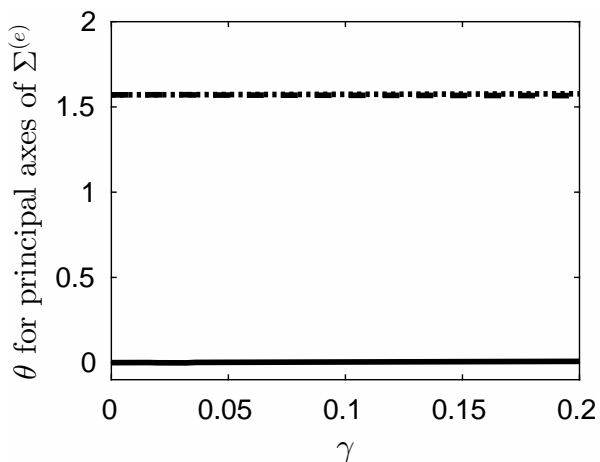

(d)

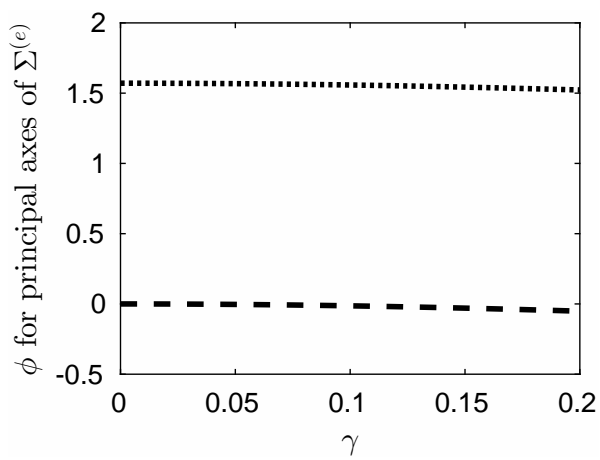

(f)

FIG. 8. (a), (b) the eigenvalues of $\Sigma^{(i)}$ and $\Sigma^{(e)} ;$ (c), (d) the $\theta$ coordinate in spherical polar coordinates of the eigenvectors of $\Sigma^{(i)}$ and $\Sigma^{(e)} ;(\mathrm{e})$, (f) the $\phi$ coordinate in spherical polar coordinates of the eigenvectors of $\Sigma^{(i)}$ and $\Sigma^{(e)}$. The second set of parameters in Section 4.3.1 was used to generate the repeating unit that we homogenise over. In all figures the solid line represents the principal component corresponding to the fibre direction, the broken line the sheet direction, and the dotted line the sheet normal direction. 


\section{Discussion}

In this study we set out to investigate the validity of two assumptions embedded within the tissue level bidomain equations: firstly the assumption that the charge on the membrane is proportional to the potential difference across the membrane; and secondly the assumption that the conductivity tensors had constant principal values across tissue, with the principal axes of the conductivity tensors being aligned with the orientation of the tissue microstructure.

To investigate the first of these assumptions we extended the model of the electrophysiology of the squid giant axon presented by Richardson (2009) that uses the Poisson-Nernst-Planck equations to model ionic concentrations and potential. We incorporated a more detailed model of the permittivity in the Debye layers given by Cherepanov et al. (2003). Provided the tissue level intracellular potential was defined to be that adjacent to the cell membrane - which may be significantly different to that away from the cell membrane - we saw that the assumption that the charge on the membrane is proportional to the potential difference across the membrane is a valid assumption for a range of membrane potentials far wider than seen under normal conditions. This definition of the intracellular potential is unlikely to cause any difficulties in practice, as the intracellular potential is usually eliminated from the tissue level bidomain equations, allowing the familiar formulation in terms of the extracellular potential and transmembrane potential. We should recall, however, that the analysis predicted changes in the potential across lengthscales that are of the same order as the size of individual ions. As a consequence we should highlight that it is possible that the continuum limit doesn't hold in these regions, and an analysis of discrete ions may be necessary.

The second asumption that we investigated was whether a simple ad hoc form of the tissue level intracellular and extracellular conductivity tensors commonly used in the bidomain equations was valid. It is usually assumed that the principal values of these tensors are constant across tissue, and that the principal directions are aligned with the microstructure, with one axis in the direction of cardiac fibres, a second axis perpendicular to the fibre but lying within the fibre sheet, and the third axis perpendicular to this sheet. Using the homogenisation technique for an almost periodic microstructure described by Richardson and Chapman (2011), and noting that the stretches usually seen in cardiac tissue are relatively small, we demonstrated that this approximation is a reasonable approximation. We did, however, illustrate that this assumption isn't always valid when larger stretches are seen, such as in cardiac tissue when a precordial thump is performed (Pellis and Kohl, 2009), and so some care must be taken when using the bidomain equations.

\section{A. Parameter values for the microscale model}

Values of some parameters that appear in the model presented in Section 2 are given in Table 1. 


\begin{tabular}{|c|c|c|}
\hline Parameter & Value & Reference \\
\hline$D_{N_{i}}^{*}$ & $7.7 \times 10^{-10} \mathrm{~m}^{2} \mathrm{~s}^{-1}$ & Niederer (2013) \\
\hline$D_{K_{i}}^{*}$ & $7.7 \times 10^{-10} \mathrm{~m}^{2} \mathrm{~s}^{-1}$ & Niederer (2013) \\
\hline$D_{C_{i}}^{*}$ & $1.5 \times 10^{-10} \mathrm{~m}^{2} \mathrm{~s}^{-1}$ & Niederer (2013) \\
\hline$D_{N_{\rho}}^{*}$ & $1.29 \times 10^{-9} \mathrm{~m}^{2} \mathrm{~s}^{-1}$ & Niederer (2013) \\
\hline$D_{K_{e}}^{*}$ & $1.29 \times 10^{-9} \mathrm{~m}^{2} \mathrm{~s}^{-1}$ & Niederer (2013) \\
\hline$D_{C_{\rho}}^{*}$ & $1.5 \times 10^{-10} \mathrm{~m}^{2} \mathrm{~s}^{-1}$ & Niederer (2013) \\
\hline$F^{e}$ & $9.65 \times 10^{4} \mathrm{C} \mathrm{mol}^{-1}$ & Richardson (2009) \\
\hline$R$ & $8.31 \mathrm{C} \mathrm{V} \mathrm{mol}^{-1} \mathrm{~K}^{-1}$ & Richardson (2009) \\
\hline$T$ & $280 \mathrm{~K}$ & Richardson (2009) \\
\hline$N_{i}^{*}$ & $4 \mathrm{~mol} \mathrm{~m}^{-3}$ & Niederer (2013) \\
\hline$K_{i}^{*}$ & $135 \mathrm{~mol} \mathrm{~m}^{-3}$ & Niederer (2013) \\
\hline$C_{i}^{*}$ & $8 \times 10^{-5} \mathrm{~mol} \mathrm{~m}^{-3}$ & Niederer (2013) \\
\hline$N_{e}^{*}$ & $140 \mathrm{~mol} \mathrm{~m}^{-3}$ & Niederer (2013) \\
\hline$K_{e}^{*}$ & $4 \mathrm{~mol} \mathrm{~m}^{-3}$ & Niederer (2013) \\
\hline$C_{e}^{*}$ & $1.2 \mathrm{~mol} \mathrm{~m}^{-3}$ & Niederer (2013) \\
\hline Membrane thickness & $7.5 \times 10^{-9} \mathrm{~m}$ & Plonsey and Barr (2000) \\
\hline$\lambda^{*}$ & $10^{-9} \mathrm{~m}$ & Cherepanov et al. (2003) \\
\hline$\varepsilon_{0}$ & $8.85 \times 10^{-12} \mathrm{~F} \mathrm{~m}^{-1}$ & Urban et al. (2013) \\
\hline \multirow[t]{3}{*}{$f^{*}$} & Between $-10^{-3} \mathrm{C} \mathrm{m}^{-3}$ & Brown and Noble (1978) \\
\hline & and $-6 \times 10^{-2} \mathrm{C} \mathrm{m}^{-3}$ & Cherepanov et al. (2003); Elul (1967) \\
\hline & & Kass and Krafte (1987); Kell and DeFelice (1988) \\
\hline
\end{tabular}

Table 1. Parameter values for the microscale model

\section{B. Proof that the conductivity tensors are symmetric and positive definite}

We first show that the intracellular conductivity tensor given by Eq. (4.9) is symmetric. Remembering that $F$ is constant over the unit we homogenise over we have

$$
\begin{aligned}
& F_{p j} \int_{\Omega_{i}^{\prime}} \frac{\partial W_{k}^{(i)}}{\partial y_{j}^{\prime}} \mathrm{d} V^{\prime} F_{k r}^{-1} \\
& =\int_{\Omega_{i}^{\prime}} F_{p j} \frac{\partial W_{k}^{(i)}}{\partial y_{j}^{\prime}} F_{k r}^{-1} \mathrm{~d} V^{\prime} \\
& =\int_{\Omega_{i}^{\prime}} F_{p j} \frac{\partial W_{k}^{(i)}}{\partial y_{j}^{\prime}} F_{k r}^{-1}+W_{k}^{(i)} F_{k r}^{-1} F_{m j} F_{m n} \frac{\partial^{2} W_{s}^{(i)}}{\partial y_{j}^{\prime} \partial y_{n}^{\prime}} F_{s p}^{-1} \mathrm{~d} V^{\prime} \text { using Eq. (4.11) } \\
& =\int_{\Omega_{i}^{\prime}} \frac{\partial}{\partial y_{j}^{\prime}}\left(F_{m s} F_{s p}^{-1} F_{m j} W_{k}^{(i)} F_{k r}^{-1}+W_{k}^{(i)} F_{k r}^{-1} F_{m j} F_{m n} F_{s p}^{-1} \frac{\partial W_{s}^{(i)}}{\partial y_{n}^{\prime}}\right)- \\
& =\int_{\partial \Omega^{\prime}} W_{k}^{(i)} F_{k r}^{-1} F_{s p}^{-1}\left(F_{m s} F_{m j} n_{j}^{\prime}+F_{m j} F_{m n} \frac{\partial W_{s}^{(i)}}{\partial y_{n}^{\prime}} n_{j}^{\prime}\right) \mathrm{d} S^{\prime}-\int_{\Omega_{i}^{\prime}} \frac{\partial W_{k}^{(i)}}{\partial y_{j}^{\prime}} F_{k r}^{-1} F_{m j} F_{m n} F_{s p}^{-1} \frac{\partial W_{s}^{(i)}}{\partial y_{n}^{\prime}} \mathrm{d} V^{\prime} \\
& =-\int_{\Omega_{i}^{\prime}} \frac{\partial W_{k}^{(i)}}{\partial y_{j}^{\prime}} F_{k r}^{-1} F_{m j} F_{m n} F_{s p}^{-1} \frac{\partial W_{s}^{(i)}}{\partial y_{n}^{\prime}} \mathrm{d} V^{\prime} \text { using Eq. (4.12). }
\end{aligned}
$$


Interchanging the indices $r$ and $p$, and repeating the argument above, allows us to deduce that

$$
F_{p j} \int_{\Omega_{i}^{\prime}} \frac{\partial W_{k}^{(i)}}{\partial y_{j}^{\prime}} \mathrm{d} V^{\prime} F_{k r}^{-1}=F_{r j} \int_{\Omega_{i}^{\prime}} \frac{\partial W_{k}^{(i)}}{\partial y_{j}^{\prime}} \mathrm{d} V^{\prime} F_{k p}^{-1} .
$$

It then follows from Eq. (4.11) that $\Sigma_{p r}^{(i)}=\Sigma_{r p}^{(i)}$, and so the intracellular conductivity tensor is symmetric. The symmetry of the extracellular conductivity tensor follows using a similar argument.

We now prove that the intracellular conductivity tensor is positive definite. Let $\mathbf{u}$ be any vector, and define the vector $\mathbf{v}$ by

$$
v_{p}=\left(\delta_{p r}+F_{p j} \frac{\partial W_{k}^{(i)}}{\partial y_{j}^{\prime}} F_{k r}^{-1}\right) u_{r}
$$

We then have

$$
\begin{aligned}
0 \leqslant & \|\mathbf{v}\|^{2} \\
= & u_{t}\left(\delta_{p t}+F_{p m} \frac{\partial W_{n}^{(i)}}{\partial y_{m}^{\prime}} F_{n t}^{-1}\right)\left(\delta_{p r}+F_{p j} \frac{\partial W_{k}^{(i)}}{\partial y_{j}^{\prime}} F_{k r}^{-1}\right) u_{r} \\
= & u_{t}\left(\delta_{t r}+F_{r m} \frac{\partial W_{n}^{(i)}}{\partial y_{m}^{\prime}} F_{n t}^{-1}+F_{t j} \frac{\partial W_{k}^{(i)}}{\partial y_{j}^{\prime}} F_{k r}^{-1}+F_{p m} \frac{\partial W_{n}^{(i)}}{\partial y_{m}^{\prime}} F_{n t}^{-1} F_{p j} \frac{\partial W_{k}^{(i)}}{\partial y_{j}^{\prime}} F_{k r}^{-1}\right) u_{r} \\
= & u_{t}\left(\delta_{t r}+F_{r m} \frac{\partial W_{n}^{(i)}}{\partial y_{m}^{\prime}} F_{n t}^{-1}+F_{k r}^{-1} F_{n t}^{-1} \frac{\partial}{\partial y_{j}^{\prime}}\left(W_{k}^{(i)} F_{a n} F_{a j}+F_{p m} F_{p j} \frac{\partial W_{n}^{(i)}}{\partial y_{m}^{\prime}} W_{k}^{(i)}\right)-\right. \\
& \left.\quad F_{k r}^{-1} F_{n t}^{-1} F_{p m} F_{p j} \frac{\partial^{2} W_{n}^{(i)}}{\partial y_{j}^{\prime} \partial y_{m}^{\prime}} W_{k}^{(i)}\right) u_{r} \\
& u_{t}\left(\delta_{t r}+F_{r m} \frac{\partial W_{n}^{(i)}}{\partial y_{m}^{\prime}} F_{n t}^{-1}+F_{k r}^{-1} F_{n t}^{-1} \frac{\partial}{\partial y_{j}^{\prime}}\left(W_{k}^{(i)} F_{a n} F_{a j}+F_{p m} F_{p j} \frac{\partial W_{n}^{(i)}}{\partial y_{m}^{\prime}} W_{k}^{(i)}\right)\right) u_{r},
\end{aligned}
$$

where we have used Eq. (4.11) in the last line above. We then integrate the final equation above over $\Omega_{i}^{\prime}$, apply the divergence theorem and the boundary condition Eq. (4.12), and obtain

$$
u_{r}\left(\int_{\Omega_{i}^{\prime}} \delta_{r t}+F_{r m} \frac{\partial W_{n}^{(i)}}{\partial y_{m}^{\prime}} F_{n t}^{-1} \mathrm{~d} V\right) u_{t} \geqslant 0
$$

and, using the definition of the intracellular conductivity tensor given by Eq. (4.9), it follows that

$$
u_{r} \Sigma_{r t}^{(i)} u_{t} \geqslant 0
$$

for all vectors $\mathbf{u}$, and so the intracellular conductivity tensor is positive definite. A similar argument can be used to show that the extracellular conductivity tensor is positive definite. 


\section{Conditions under which rotation matrices and diagonal matrices commute}

Suppose $P$ is a $3 \times 3$ orthonormal matrix with $\operatorname{det}(P)=1, D$ is a $3 \times 3$ diagonal matrix, and that $P$ and $D$ have entries given by

$$
P=\left(\begin{array}{lll}
P_{11} & P_{12} & P_{13} \\
P_{21} & P_{22} & P_{23} \\
P_{31} & P_{32} & P_{33}
\end{array}\right), \quad D=\left(\begin{array}{ccc}
D_{11} & 0 & \\
0 & D_{22} & 0 \\
0 & 0 & D_{33}
\end{array}\right)
$$

where the diagonal entries of $D$ are non-zero. Suppose the matrices $P$ and $D$ commute, i.e. $P D=D P$. We then have $P=D^{-1} P D$, and so

$$
\left(\begin{array}{lll}
P_{11} & P_{12} & P_{13} \\
P_{21} & P_{22} & P_{23} \\
P_{31} & P_{32} & P_{33}
\end{array}\right)=\left(\begin{array}{ccc}
P_{11} & \frac{D_{22}}{D_{11}} P_{12} & \frac{D_{33}}{D_{11}} P_{13} \\
\frac{D_{11}}{D_{22}} P_{21} & P_{22} & \frac{D_{33}}{D_{22}} P_{23} \\
\frac{D_{11}}{D_{33}} P_{31} & \frac{D_{22}}{D_{33}} P_{32} & P_{33}
\end{array}\right) .
$$

This equation is clearly satisfied if $D_{11}=D_{22}=D_{33}$, i.e. $D$ is a multiple of the identity matrix. Suppose $D$ is not a multiple of the identity matrix and $D_{11} \neq D_{22}$. Clearly from the matrix equation above we must have $P_{12}=P_{21}=0$. Furthermore we see that if one of $P_{13}$ and $P_{31}$ is non-zero we would require $D_{11}=D_{33}$, and if one of $P_{23}$ and $P_{32}$ is non-zero we would require $D_{22}=D_{33}$ - the combination of these would then require $D_{11}=D_{22}$, which would contradict $D_{11} \neq D_{22}$.

One way to allow $P$ and $D$ to commute, with $D_{11} \neq D_{22}$, is to set $P_{12}=P_{21}=P_{13}=P_{31}=0$. Allowing $P_{23}$ and $P_{32}$ to take non-zero values, we have $D_{22}=D_{33}$. The entries of $P$ and $D$ are then given by

$$
P=\left(\begin{array}{ccc}
P_{11} & 0 & 0 \\
0 & P_{22} & P_{23} \\
0 & P_{32} & P_{33}
\end{array}\right), \quad D=\left(\begin{array}{ccc}
D_{11} & 0 & \\
0 & D_{22} & 0 \\
0 & 0 & D_{22}
\end{array}\right)
$$

Remembering that $P$ is an orthonormal matrix with $\operatorname{det}(P)=1$ we may write the entries of $P$ as

$$
P=\left(\begin{array}{ccc}
1 & 0 & 0 \\
0 & \cos \theta & -\sin \theta \\
0 & \sin \theta & \cos \theta
\end{array}\right)
$$

We then see that $P$ represents a rotation with axis along the first coordinate direction, and that the stretches represented by the diagonal matrix are identical in the coordinate directions perpendicular to the axis of rotation. All other combinations of $P$ and $D$ that commute follow this pattern - the axis of rotation is along one of the coordinate axes, and the stretches are equal in the directions perpendicular to this axis.

\section{References}

Beeler, G. and Reuter, H. (1977). Reconstruction of the action potential of ventricular myocardial fibres. Journal of Physiology, 268:177-210.

Bishop, M., Rodriguez, B., Qu, F., Efimov, I., Gavaghan, D., and Trayanova, N. (2007). The role of photon scattering in optical signal distortion during arrhythmia and defibrillation. Biophysical Journal, 93:3714-3726. 
Brown, R. and Noble, D. (1978). Displacement of activation thresholds in cardiac muscle by protons and calcium ions. Journal of Physiology, 282:333-343.

Bruce, D., Pathmanathan, P., and Whiteley, J. (2014). Modelling the effect of gap junctions on tissuelevel cardiac electrophysiology. Bulletin of Mathematical Biology, 76:431-454.

Cherepanov, D., Feniouk, B., Junge, W., and Mulkidjanian, A. (2003). Low dielectric permittivity of water at the membrane interface: effect on the energy coupling mechanism in biological membranes. Biophysical Journal, 85:1307-1316.

Clerc, L. (1976). Directional differences of impulse spread in trabecular muscle from mammalian heart. Journal of Physiology, 255:335-346.

Ebihara, L. and Johnson, E. (1980). Fast sodium current in cardiac muscle. Biophysical Journal, 32:779-790.

Elul, R. (1967). Fixed charge in the cell membrane. Journal of Physiology, 189:351-365.

Guyton, A. and Hall, J. (1996). Textbook of Medical Physiology. W.B. Saunders, Philadelphia, USA.

Hand, P. and Griffith, B. (2010). Adaptive multiscale model for simulating cardiac conduction. Proceedings of the National Academy of Sciences of the United States of America, 107:14603-14608.

Hand, P. and Griffith, B. (2011). Empirical study of an adaptive multiscale model for simulating cardiac conduction. Bulletin of Mathematical Biology, 73:3071-3089.

Hunter, P., McCulloch, A., and ter Keurs, H. (1998). Modelling the mechanical properties of cardiac tissue. Progress in Biophysics and Molecular Biology, 69:289-331.

Kass, R. and Krafte, D. (1987). Negative surface charge density near heart calcium channels. Journal of General Physiology, 89:629-644.

Keener, J. and Panfilov, A. (1996). A biophysical model for defibrillation of cardiac tissue. Biophysical Journal, 71:1335-1345.

Keener, J. and Sneyd, J. (1998). Mathematical Physiology. Springer, New York, USA.

Kell, M. and DeFelice, L. (1988). Surface charge near the cardiac inward-rectifier channel measured from single-channel conductance. Journal of Membrane Biology, 102:1-10.

Luo, C. and Rudy, Y. (1994). A dynamic model of the cardiac ventricular action potential. I. Simulations of ionic currents and concentration changes. Circulation Research, 74:1071-96.

Neu, J. and Krassowska, W. (1993). Homogenization of syncytial tissues. Critical Reviews in Biomedical Engineering, 21:137-199.

Newman, J. and Thomas-Alyea, K. (2004). Electrochemical Systems. Wiley, New Jersey, USA.

Niederer, S. (2013). Regulation of ion gradients across myocardial ischemic border zones: a biophysical modelling analysis. PLOS One, 8:e60323.

Niederer, S., Hunter, P., and Smith, N. (2006). A quantitative analysis of cardiac myocyte relaxation: a simulation study. Biophysical Journal, 90:1697-1722. 
Noble, D. and Rudy, Y. (2001). Models of cardiac ventricular action potentials: iterative interaction between experiment and simulation. Philosophical Transactions of the Royal Society of London A, 359:1127-1142.

Pellis, T. and Kohl, P. (2009). Extracorporeal cardiac mechanical stimulation: precordial thump and precordial precision. British Medical Bulletin, 93:161-177.

Plonsey, R. and Barr, R. C. (2000). Bioelectricity, A Quantitative Approach. Kluwer Academic / Plenum Publishers, New York, USA.

Richardson, G. (2009). A multiscale approach to modelling electrochemical processes occurring across the cell membrane with application to transmission of action potentials. Mathematical Medicine and Biology, 26:201-224.

Richardson, G. and Chapman, S. (2011). Derivation of the bidomain equations for a beating heart with general microstructure. SIAM Journal on Applied Mathematics, 71:657-675.

Sommer, J. (1983). Implications of structure and geometry on cardiac electrical activity. Annals of Biomedical Engineering, 11:149-157.

Teschke, O., Ceotto, G., and de Souza, E. (2001). Interfacial water dielectric-permittivity-profile measurements using atomic force microscopy. Physical Review E, 64:011605.

Trefethen, L. and Bau, D. (1997). Numerical Linear Algebra. SIAM, Philadelphia, USA.

Urban, M., Couchot, F., Sarazin, X., and Djannati-Atai, A. (2013). The quantum vacuum as the origin of the speed of light. The European Physical Journal D, 67:58. 


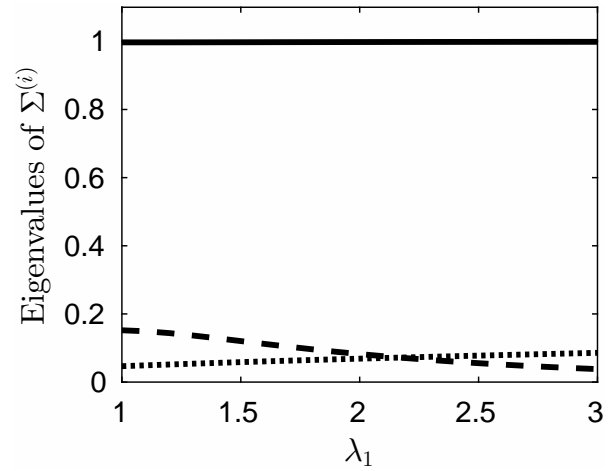

(a)

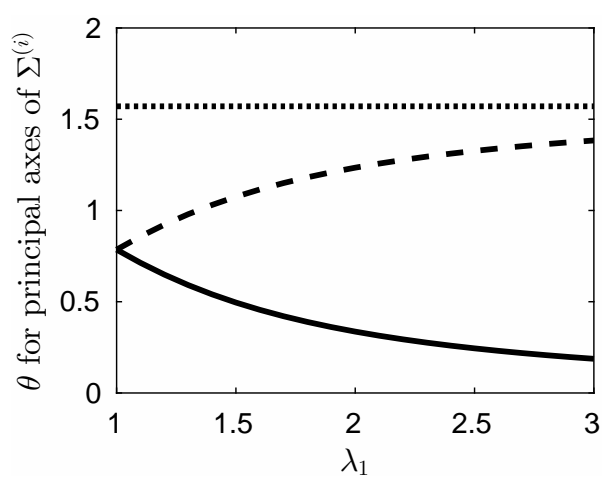

(c)

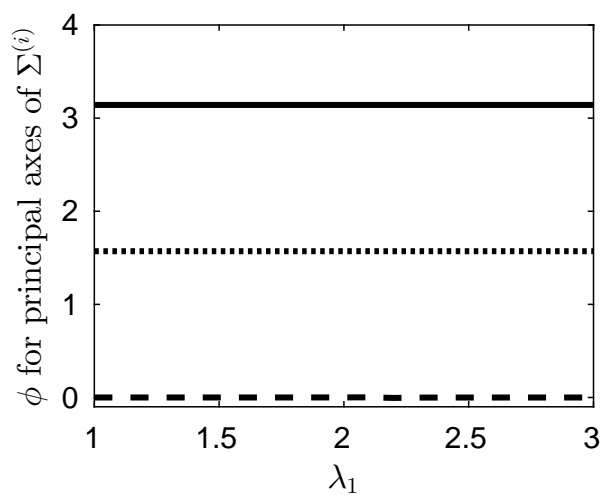

(e)

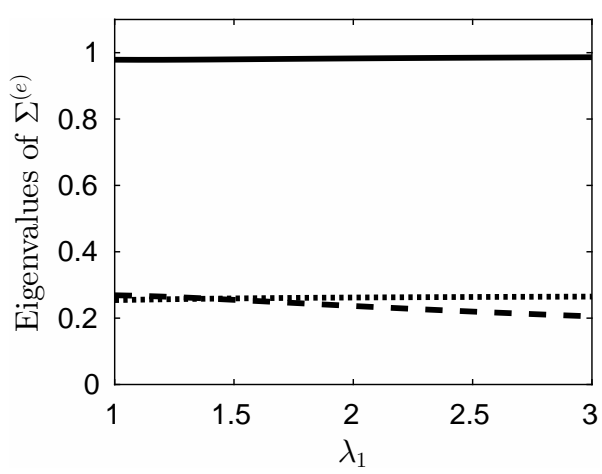

(b)

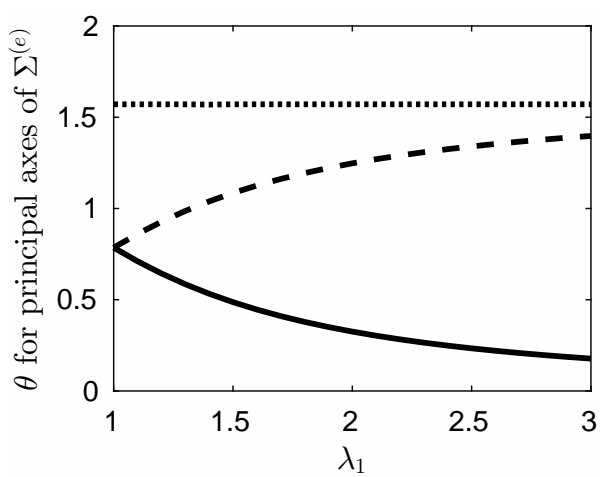

(d)

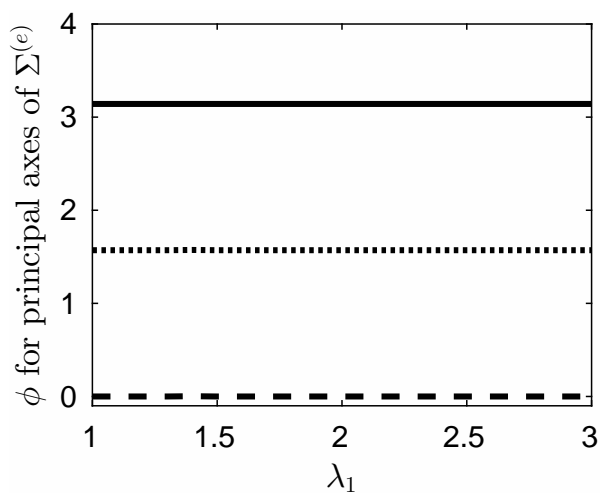

(f)

FIG. 9. (a), (b) the eigenvalues of $\Sigma^{(i)}$ and $\Sigma^{(e)} ;$ (c), (d) the $\theta$ coordinate in spherical polar coordinates of the eigenvectors of $\Sigma^{(i)}$ and $\Sigma^{(e)} ;(\mathrm{e})$, (f) the $\phi$ coordinate in spherical polar coordinates of the eigenvectors of $\Sigma^{(i)}$ and $\Sigma^{(e)}$. The second set of parameters in Section 4.3.1 was used to generate the repeating unit that we homogenise over. In all figures the solid line represents the principal component corresponding to the fibre direction in the unit we homogenise over, the broken line the sheet direction, and the dotted line the sheet normal direction. 